\title{
Sonic Hedgehog repression underlies gigaxonin mutation-induced motor deficits in giant axonal neuropathy
}

\author{
Yoan Arribat, ${ }^{1}$ Karolina S. Mysiak, ${ }^{1}$ Léa Lescouzères, ${ }^{1}$ Alexia Boizot, ${ }^{1}$ Maxime Ruiz, ${ }^{1}$ Mireille Rossel, ${ }^{2}$ and Pascale Bomont ${ }^{1}$ \\ 'ATIP-Avenir team, INM, INSERM, University of Montpellier, Montpellier, France. ${ }^{2}$ MMDN, University of Montpellier, EPHE, INSERM, U1198, PSL Research University, Montpellier, France.
}

\begin{abstract}
Growing evidence shows that alterations occurring at early developmental stages contribute to symptoms manifested in adulthood in the setting of neurodegenerative diseases. Here, we studied the molecular mechanisms causing giant axonal neuropathy (CAN), a severe neurodegenerative disease due to loss-of-function of the gigaxonin-E3 ligase. We showed that gigaxonin governs Sonic Hedgehog (Shh) induction, the developmental pathway patterning the dorso-ventral axis of the neural tube and muscles, by controlling the degradation of the Shh-bound Patched receptor. Similar to Shh inhibition, repression of gigaxonin in zebrafish impaired motor neuron specification and somitogenesis and abolished neuromuscular junction formation and locomotion. Shh signaling was impaired in gigaxonin-null zebrafish and was corrected by both pharmacological activation of the Shh pathway and human gigaxonin, pointing to an evolutionary-conserved mechanism regulating Shh signaling. Gigaxonin-dependent inhibition of Shh activation was also demonstrated in primary fibroblasts from patients with GAN and in a Shh activity reporter line depleted in gigaxonin. Our findings establish gigaxonin as a key E3 ligase that positively controls the initiation of Shh transduction, and reveal the causal role of Shh dysfunction in motor deficits, thus highlighting the developmental origin of GAN.
\end{abstract}

\section{Introduction}

The concept of developmental defects in neurodegenerative diseases is well established for early-onset conditions, and is now emerging for late-onset pathologies where symptoms only become detectable in adulthood. Diseases presenting with cortical malformations, such as infantile epilepsy, Rett syndrome, mental retardation, and autism are all considered developmental diseases. Indeed, the corresponding genes have been shown to control critical functions for neuronal development, encompassing neuronal patterning, proliferation, migration, and synaptogenesis. For adult pathologies, such as Alzheimer's (AD) and Huntington's (HD) diseases, the hypothesis of a developmental signature has been supported by experimental and clinical studies, revealing the expression of $\beta$-amyloid precursor protein (APP) and Huntingtin (Htt) gene products (1-3), respectively, during developmental stages, and alterations of the cortex morphology in presymptomatic $\mathrm{AD}$ and $\mathrm{HD}$ patients using magnetic resonance imaging (MRI) $(4,5)$. Growing evidence shows that genes mutated in late-onset diseases play essential roles during development, such as cortical progenitor migration for APP and axonal transport for $\mathrm{Htt}(6,7)$. Furthermore, studies on mouse models reveal various alterations

Authorship note: YA and KSM are co-first authors and contributed equally to the present work.

Conflict of interest: The authors have declared that no conflict of interest exists. Copyright: (5) 2019, American Society for Clinical Investigation.

Submitted: April 30, 2019; Accepted: August 28, 2019; Published: October 28, 2019

Reference information: / Clin Invest. 2019;129(12):5312-5326.

https://doi.org/10.1172/JCI129788. of neurodevelopmental processes: altered morphology of cranial nerves and truncated lumbar spinal nerves in spinal muscular atrophy embryos (8); impairment of striatal neuron specification and maturation (9); and alteration of cortical progenitor cell division and neurogenesis (10) in HD embryos. This culminated with the direct demonstration of the implication of neurodevelopmental deficits in HD pathogenesis, in a study where mice expressing mutant Htt solely during development recapitulate the human disease (11) from the striatal neurodegeneration to the electrophysiological and motor performance deficits.

Considering that late-onset pathologies can have an origin during development, it would be reasonable to hypothesize that this is also the case for early-onset diseases. Among those, the infantile and fatal neurodegenerative disease giant axonal neuropathy (GAN) (12) represents a strong candidate. Around 2 years of age, children with GAN become rapidly challenged in both motor and sensory modalities $(13,14)$. From an initial decrease of deep tendon reflexes, areflexia, and amyotrophy, patients rapidly evolve toward a total loss of deep and superficial sensitivity and of ambulation, and become wheelchair-bound during adolescence. The dramatic deterioration of the peripheral nervous system subsequently spreads to the central nervous system (CNS), causing numerous symptoms encompassing ataxia, dysarthria, nystagmus, vision impairment, intellectual disabilities, and epilepsy in young adults. Altogether, the sum of symptoms leading to the death of patients in the third decade of life is incredibly extensive, and distinguishes GAN from other neurodegenerative disorders, for which a regionalization of neuronal deficits and neurodegeneration is observed. Altogether, one can presume a fundamental role 
of gigaxonin (15), the defective protein in GAN, in controlling a general pathway to sustain neuronal function. It is noteworthy that brain MRI of patients has revealed atrophy of the spinal cord, cerebellum and the brain stem, which would favor a global defect in brain development over a progressive neurodegeneration (16). In line with this hypothesis, a morphological marker of developmental deficit (cavum septi pellucidi) has been evidenced in numerous patients with GAN (16). Finally, while gigaxonin expression pattern has been shown to be ubiquitous, it was found to be enriched in the nervous system and during prenatal stages (17), which may suggest a role during embryogenesis.

Our understanding of the general mode of action of gigaxonin emerges from its domain characterization as a BTB-Kelch protein (15) and its identification as an adaptor of Cul3-E3 ubiquitin ligase complex (18). In disease, the discovery that GAN mutations, which are scattered along the entire GAN gene, confer a general instability of either the mRNA or the protein (19), supports a loss-offunction of the gigaxonin-E3 ligase in patients. So far, the mostestablished substrates of gigaxonin are the intermediate filament (IF) cytoskeletal proteins (20), due to the fact that they represent a hallmark of the disease and can be easily investigated in patient skin-derived fibroblasts. Thus, the broad aggregation of IFs in neuronal (neurofilaments) and nonneuronal tissues in patients (21) has been studied in patient-derived fibroblasts, GAN mice $(17,22)$, and their derived neuronal models (23). According to its putative role as E3 ligase adaptor, gigaxonin imbalance was shown to either induce a dramatic clearance of multiple IF types upon excess (23, 24) or an abnormal aggregation upon depletion (25-27). The study of the GAN neuronal model also unveiled the fundamental role of gigaxonin in controlling the autophagy pathway by regulating the production of autophagosomes through the ubiquitin-dependent degradation of the ATG16L1 protein (28). While both neurofilaments and the ATG16L1 autophagy protein have been identified as targets of gigaxonin, their respective contribution(s) to neuronal impairment and neurodegeneration in GAN remain(s) to be determined. In this context, revealing the molecular mechanisms controlled by gigaxonin is crucial.

Presently, our knowledge on the GAN pathogenesis is poor, and was mostly hampered by our inability to reproduce the severity of symptoms in the mouse $(17,22)$. In the present study, we generated a robust animal model of GAN in zebrafish, exhibiting the severity and penetrance of the motor deficits seen in patients. Furthermore, we combined its physiological analysis with biochemical data and studies on patient-derived cellular models to identify a substantial developmental signature in the pathogenesis of GAN, which originates from the control of Sonic Hedgehog (Shh) induction by the gigaxonin-E3 ligase.

The Hedgehog family of morphogens represents an evolutionarily conserved pathway essential for embryonic development, tissue homeostasis, and tumorigenesis (29). In vertebrates, Shh assigns neuronal and muscle fate, acting in a graded manner to pattern the dorso-ventral axis of the neural tube (30) and the muscles (31). Dysregulation of the Shh cascade causes a wide range of human diseases, including congenital malformations of the CNS, of the axial skeleton and limbs, cancers, and malignancies in children and adults $(32,33)$. The morphogen Shh is expressed and is cleaved in the notochord and the floor plate, releasing an
N-terminal active fragment, which diffuses to the receiving tissues. In progenitor cells, Shh initiates signaling by binding to the transmembrane receptor Patched (Ptch), thereby relieving the constitutive inhibition of another transmembrane protein, Smoothened (Smo), and allowing its accumulation on the cell surface (34). Activated Smo transduces Sh signal by inducing the nuclear translocation and the activation of Ci/Gli transcription factors to trigger the expression of patterning and differentiation genes. In vertebrates, the components of the Shh pathway are localized to the primary cilium of the cell, which is an essential organelle for its transduction. The disruption of ciliary components alters Shh activity (35) in a tissue-dependent manner, either promoting or inhibiting signal transduction $(36,37)$.

Little is known about how Shh activity is fine-tuned, but ubiquitination has emerged as an important mechanism. The degradation of Shh components by ubiquitination is key in regulating the signaling pathway. In particular, the ubiquitination of $\mathrm{Ci} / \mathrm{Gli}$ and its regulatory complex has been shown to play a pivotal role in providing a negative feedback loop for downstream Shh signaling (38). In the absence of the morphogen, partial degradation mediated by the Slimb/Trcp-E3 ligases converts Ci/Gli into a transcriptional repressor, whereas the Shh cascade is turned off after the complete degradation of active Ci/Gli by the HIB/Spop/ Itch E3 ligases (38). Ubr3-E3 ligase acts in a positive feedback loop to degrade Cos2/Kif7, a negative regulator of the Hh pathway (39), while the MIDI E3 ligase has been shown to mediate the ubiquitin-dependent cleavage of Sufu, another negative regulator of the Ci/Gli activity (40). On the other hand, the deubiquitinase Usp7/HAUSP positively regulates signal transduction by stabilizing Ci/Gli (41), which suggests a very balanced relationship between ubiquitination and deubiquitination to control Shh activity. Although the downstream Shh cascade has been well studied, less is known about how the Ptch receptor, the entry point of Shh signaling, is regulated. The Smurf-E3 ligase acts on the unbound Ptch as a mechanism to turn off Shh activity $(42,43)$, and the E3 ligase Itchy regulates the basal turnover of Ptch in the absence of the morphogen, to control the noncanonical Hh signaling (44). Thus, while the last decade has shown that ubiquitination serves as a pivotal mechanism in regulating Shh activity, the identity of the E3 ligase(s) that control(s) Ptch degradation in the presence of the morphogen, necessary for the derepression of the signal transducer Smo, is unknown.

Here, we identify gigaxonin as the E3 ligase controlling the initial steps of Shh induction, which is sufficient to specify neuronal and muscle fate in vertebrates. Using the zebrafish as a model system, we provide physiological evidence to show that the gigaxonin-E3 ligase is a key regulator of Shh activation, by controlling the degradation of the Ptch receptor in a Shh-dependent manner. Both transient and genetic repression of gigaxonin impairs spinal motor neuron specification and abolishes motility in zebrafish. The deficits of the gigaxonin-null embryos reproduce numerous animal models of Shh inhibition and are restored upon Shh activation. The positive control of gigaxonin on the pathway is Shh-dependent, as revealed independently in the gigaxonin-depleted zebrafish, in a cellular system using a Shh activity reporter assay, and in patient cells. Furthermore, gigaxonin interacts with Ptch and mediates its degradation in a Shh-dependent manner, hence identifying gigax- 


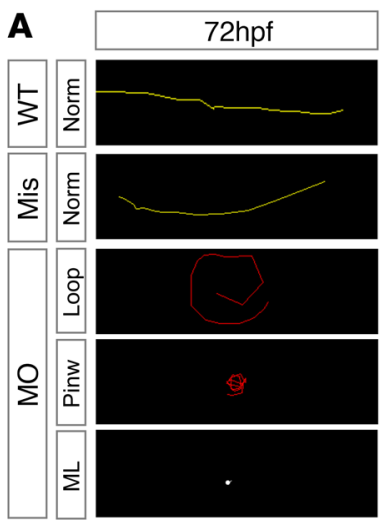

\section{B}
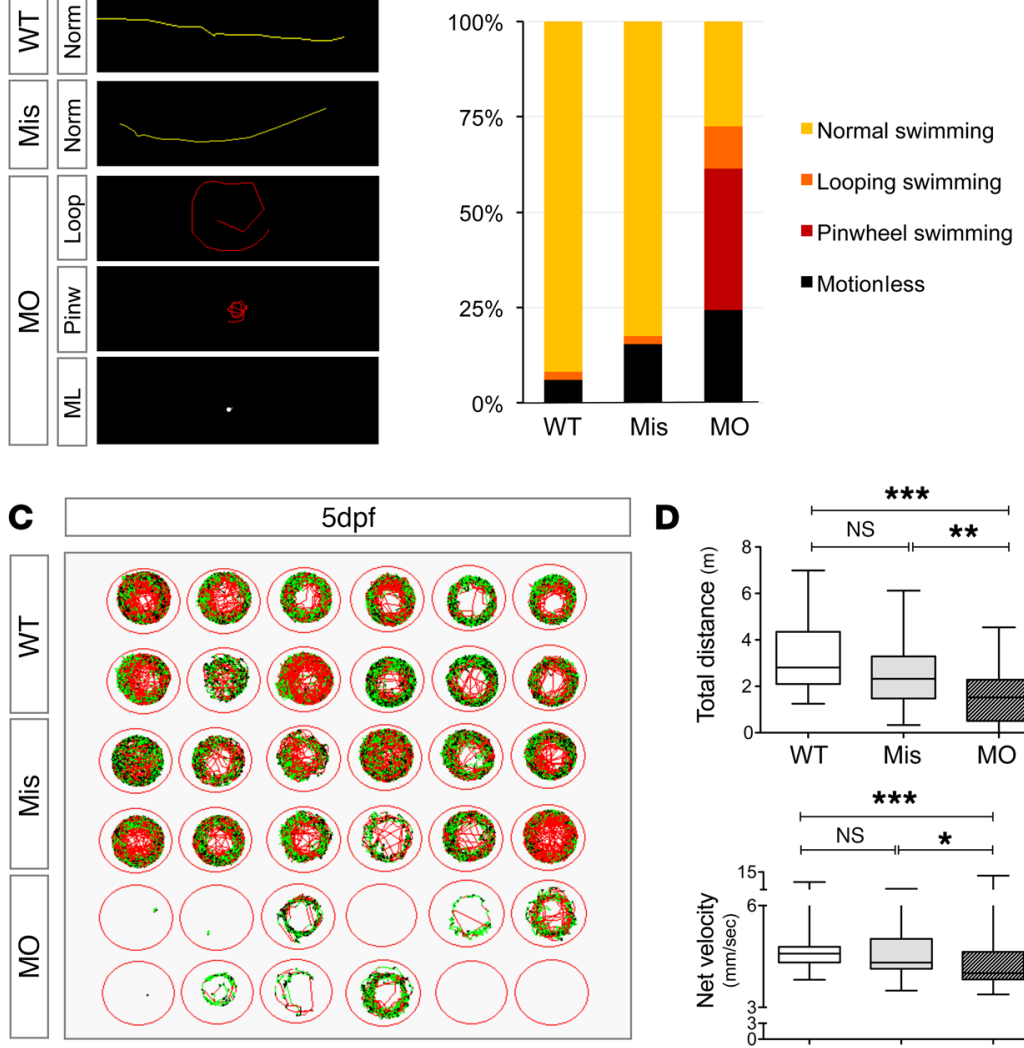

- High velocity: $6 \mathrm{~mm} / \mathrm{sec}<\mathrm{v}$

- Slow velocity: $3 \mathrm{~mm} / \mathrm{sec}<\mathrm{v}<6 \mathrm{~mm} / \mathrm{sec}$

Figure 1. Loss of motility in gan morphants. (A) WT and Mis- and MO-injected embryos were examined using the touch-response test at $72 \mathrm{hpf}$. The swimming pattern was recorded as normal, motionless (ML, absence of response), looping (Loop, circular trajectory), and pinwheel (Pinw) (see Supplemental Video 1). (B) Quantification of the percentage of embryos exhibiting the different motor behaviors in the control $(n=139)$, Mis $(n=66)$, and MO $(n=60)$ populations. (C) Tracking analysis of the spontaneous locomotion at $5 \mathrm{dpf}$. Red and green trajectories correspond to fast and slow swimming, respectively. Motility is abolished in $80 \%$ MO-injected larvae. (D) Quantification of the covered distance (top panel) and net velocity (lower panel) during spontaneous locomotion at $5 \mathrm{dpf}$. Statistics: in the absence of normality of distribution of the data, a Kruskal-Wallis test (Dunn's post hoc test) was applied; medians with interquartile range, minimum, and maximum values are represented; $n=48$ (WT), $n=48$ (Mis), $n=49$ (MO); NS, not statistically significant; ${ }^{*} P<0.05,{ }^{* *} P<0.01,{ }^{* * *} P<0.001$.

onin as key regulator of the initiation of Shh signaling. Notably, our findings obtained in the GAN zebrafish model mimic the motor dysfunctions found in patients, hence providing the first hints, to our knowledge, into the pathophysiological mechanisms in GAN and supporting a developmental origin in the pathogenesis of GAN. This notion is further endorsed by the functional rescue of the developmental deficits in the gan zebrafish by the human gigaxonin, and the evidence of an impairment of Shh signaling in patient cells.

\section{Results}

Identification and expression pattern of gigaxonin in zebrafish. In the present study, we identified a unique gigaxonin ortholog in zebrafish by bioinformatic analysis. Extension of the partial coding sequences from the zebrafish (XM003200434, AL923051, and GO940773) allowed us to clone the full-length gan cDNA (released to Genbank under accession numbers KT013299 and ANJ65950). Similar to humans, the zebrafish gan gene is composed of 11 exons and encodes for a protein of 609 amino acids with a N-terminal BTB domain and a 6 Kelch repeat domain in its C-terminal part (Supplemental Figure 1; supplemental material available online with this article; https://doi. org/10.1172/JCI129788DS1; see complete unedited blots in the supplemental material). Zebrafish gigaxonin (z-gigaxonin) is closely related to its mammalian homologs and exhibits a high degree of conservation with the human protein (78\% identity and $80 \%$ similarity) (Supplemental Figure 1).

To determine the expression pattern of z-gigaxonin, we combined whole-mount in situ hybridization with reverse transcription PCR (RT-PCR) experiments. Analysis of gan transcripts revealed ubiquitous and constant expression from early embryogenesis through juvenile/adult stages (Supplemental Figure 2, A and C, left panel), with enrichment in the eyes, the notochord, the muscles, and the heart (Supplemental Figure 2A). Interestingly, the detection of gan mRNA at the zygote stage demonstrates that maternal transcripts are present in the embryos, before the midblastula transition.

Gigaxonin depletion causes severe morphological abnormalities. To investigate the function of gigaxonin in zebrafish development, we first took a transient repression approach to enable a dose-response analysis. Indeed, we intended to achieve a significant decrease of z-gigaxonin abundance, without totally abolishing it, to more closely mimic the spectrum of GAN mutations in patients, for which a general mechanism of instability was demonstrated (19). Thus, we impaired gan pre-mRNA splicing with antisense morpholino oligonucleotide (MO), at the acceptor splice site of exon 3 (Supplemental Figure 2B). The effective disruption of splicing was confirmed by RT-PCR in morpholino-injected embryos (called gan morphants) from $10 \mathrm{hpf}$ and from a dose of $0.25 \mathrm{pmol}$ of gan oligonucleotides (Supplemental Figure 2C, right panel). Importantly, increasing doses of morpholino were compared to identify $0.25 \mathrm{pmol}$ as a nontoxic dose that did not perturb the global development and the number of somites at $24 \mathrm{hpf}$, as compared with noninjected embryos (Supplemental Figure 2D).

Injection of gan morpholino, and not the 5-bp mismatch control morpholino (Mis), induced significant abnormalities from $48 \mathrm{hpf}$, as revealed by H\&E staining of whole embryos (Supplemental Figure 3A). Morphants exhibited penetrant and strong morphological phenotypes, including shortened body length, absent yolk extension, pronounced head and eye atrophies, and heart defect (Supplemental Figure 3B). Importantly, the developmental deficits of gan morphants were rescued by coinjection of human GAN mRNA, hence confirming the specificity of the morpholino and the functional conservation of gigaxonin between zebrafish and human (Supplemental Figure 4A). Additionally, we disrupted gan mRNA processing with another 
A

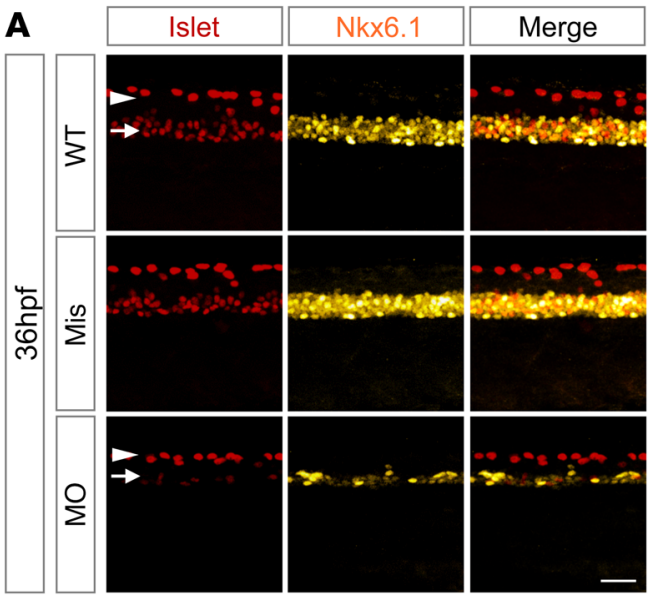

C

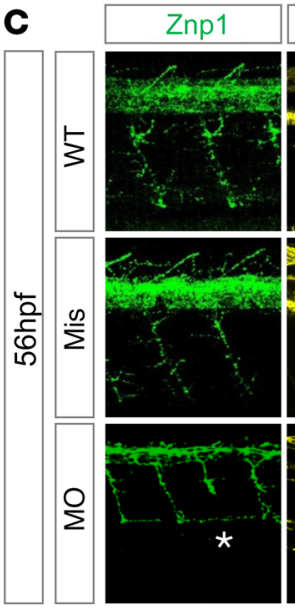

$\mathrm{Zn} 8$

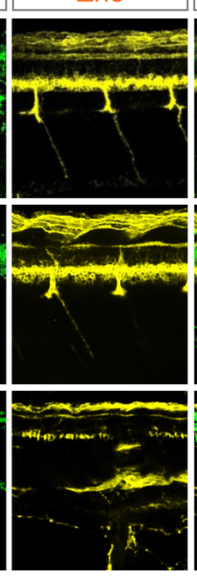

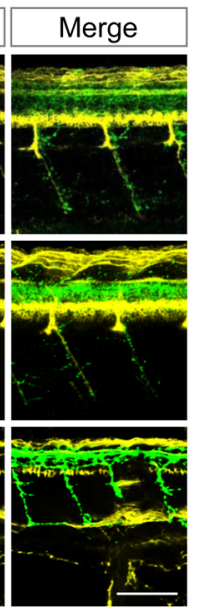

B

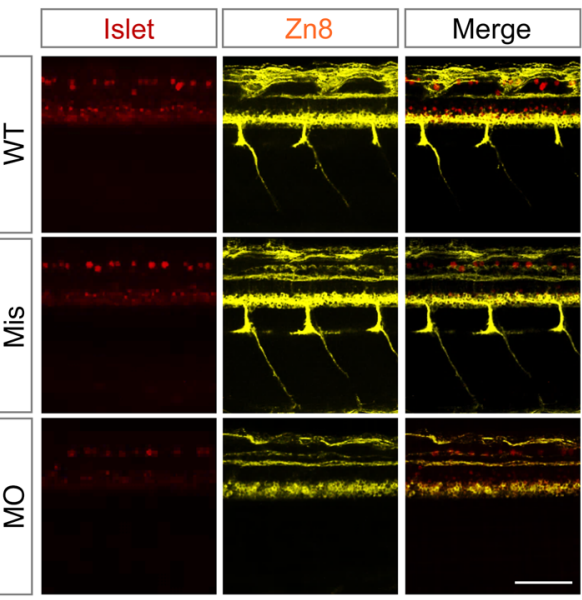

D

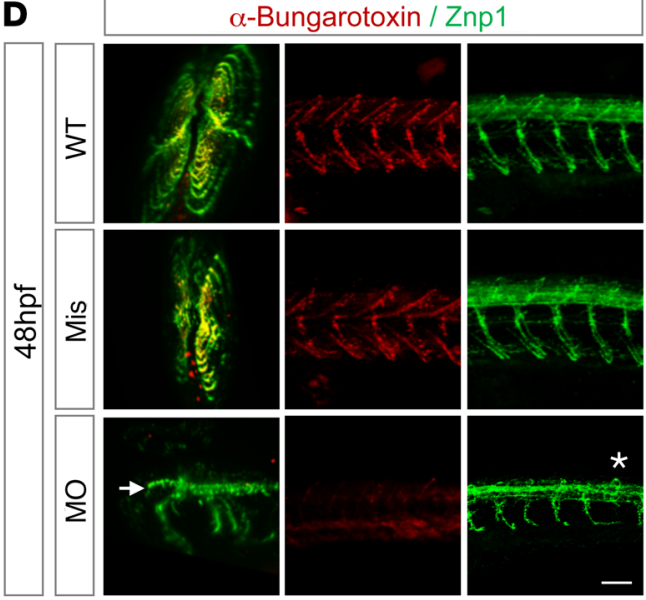

E
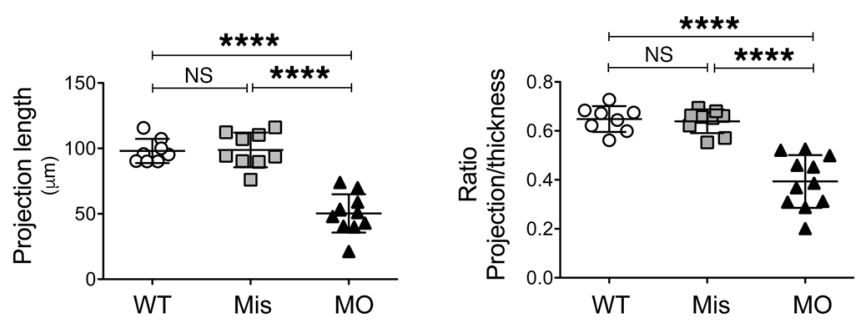

Figure 2. Impaired MN specification, altered axonogenesis, and neuromuscular junctions in gan morphants. (A) Gigaxonin repression impairs late specification of motor neuron progenitors and differentiation of sMNs. Progenitors and motor neurons were visualized with Nkx6.1 and Islet immunostaining, respectively, in a lateral view of spinal cord in WT and Mis-, and MO-injected embryos at 36 hpf. Ventral Islet-positive cells correspond to motor neurons (arrow), dorsal Isletpositive cells correspond to the sensory Rohon-Beard neurons (arrowhead). In the absence of gigaxonin, a large reduction in both Nkx6.1-positive MN progenitors and Islet-positive sMN is observed at $36 \mathrm{hpf}$ (B-E) Gigaxonin depletion leads to abnormal architecture of MN axons. (B) Gigaxonin depletion leads to the absence of sMN cell bodies (Islet) and axons (Zn8) at 56 hpf. (C) pMN axonal projections (Znp1) show abnormal arborization in morphants at 56 hpf, with some CaP pMN axons exhibiting reduced length (white asterisk). (D, left) Three-dimensional examination of the spinal cord of gigaxonin-depleted embryos at $48 \mathrm{hpf}$, using LightSheet microscopy. Gigaxonin depletion leads to additional abnormalities in the spinal cord architecture, such as protruding axons (arrow) and absence of MiP and RoP motor axons (transverse view) (see Supplemental Videos 2 and 3). (D, middle and right) Neuromuscular junctions ( $\alpha$-bungarotoxin) and subsequent innervation of trunk muscles are abolished in gan morphants. Note that CaP axons are occasionally absent (white asterisk). (E) Quantification of the mean length of pMN CaP axons (left) and mean length relative to the body thickness of the embryos, as defined by phase contrast pictures (right), showing significant neurite abnormalities. Statistics: with normality of the distribution of the data, a 1-way ANOVA test (Bonferroni's post hoc test) was used. Data represent mean \pm SD; individual values are represented; $n=8$ (WT), $n=9$ (Mis), $n=11$ (MO); NS, not statistically significant; ${ }^{* * *} P<0.0001$. Scale bars: $25 \mu \mathrm{m}$ (A); $100 \mu \mathrm{m}$ (B-D).

MO, targeting the acceptor splice site of exon 2 (MO ex1-2), and obtained similar morphological deficits (data not shown).

Gigaxonin depletion impairs motility in zebrafish. In regard to the neuronal and muscle expression of gigaxonin during development, we investigated the motor performances of the gan morphants upon touch stimulation and during spontaneous motility. The anal- ysis revealed a marked reduction of motility in gigaxonin-depleted animals. The touch-response assay, performed at $72 \mathrm{hpf}$, revealed swimming abnormalities in $72.4 \%$ of the morphants, with a circular swim (referred to as looping), circular swim around the axis of the head (referred to as pinwheel), or absence of motion (referred to as motionless) (Figure 1, A and B and Supplemental Video 1). 
A
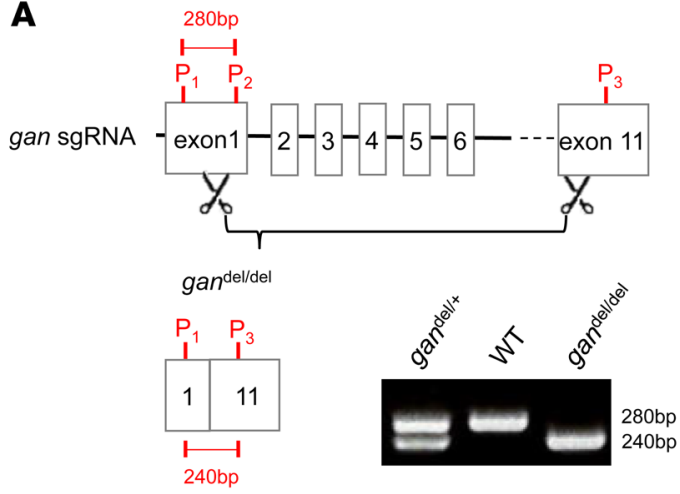

C

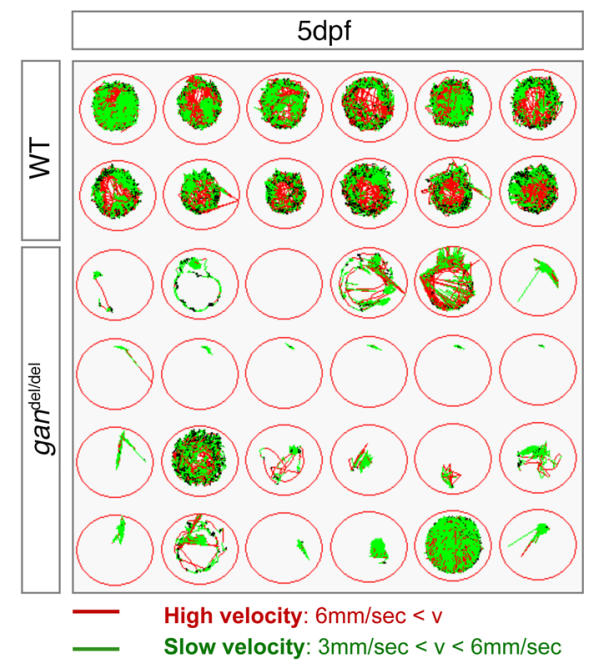

B

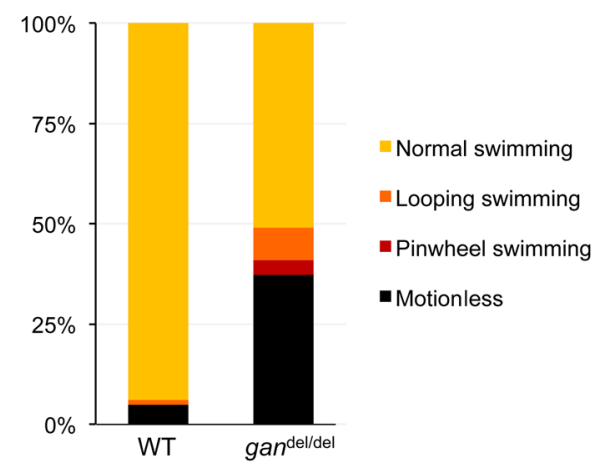

D
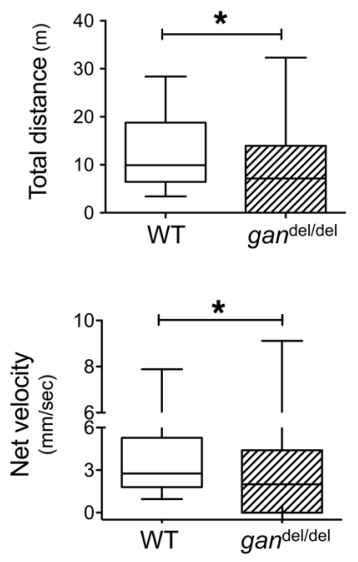
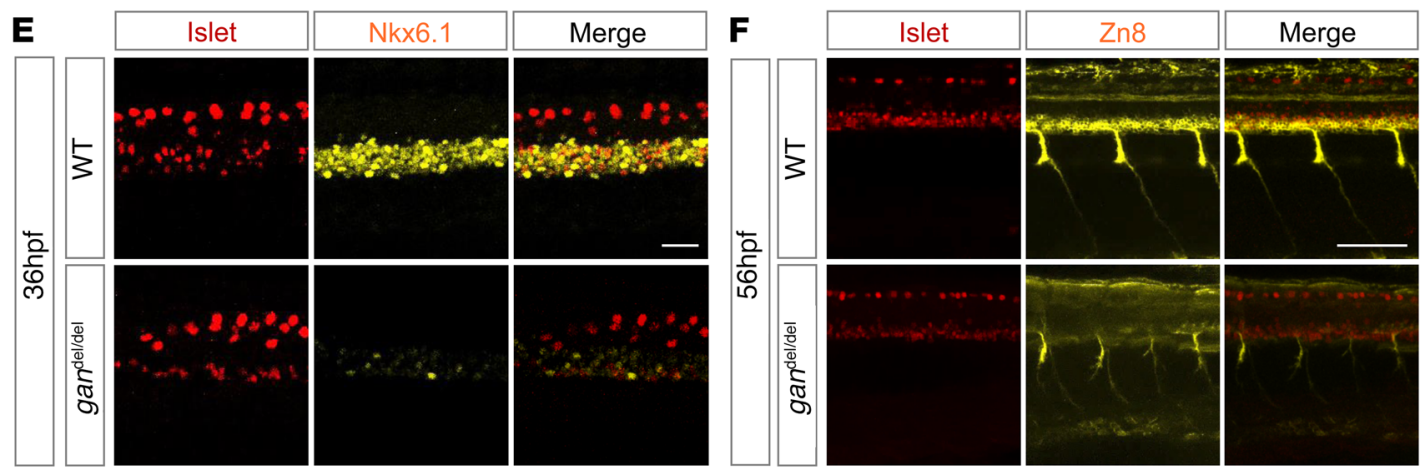

Figure 3. The gan $^{\text {del/del }}$ zebrafish, similar to gan morphants, exhibit Shh-like deficits. (A) Schematic representation of the deletion within the endogenous gan locus, and genotyping of the gan del/del line. (B-D) gan del/del embryos display locomotor deficits. (B) Quantification of the touch-evoked response of gan ${ }^{\text {del/del }}$ embryos at $72 \mathrm{hpf} ; n=82$ (WT), $n=110$ ( $\left.\mathrm{gan} \mathrm{n}^{\mathrm{del} / \mathrm{del}}\right)$. (C) Tracking analysis of the spontaneous locomotion of $5 \mathrm{dpf}$ control and mutant larvae. (D) Quantification of total distance traveled (top) and net velocity (bottom) at $5 \mathrm{dpf}$ in the control $(n=24)$ and gan ${ }^{\text {del/del }}(n=64)$ populations. Statistics: in the absence of normality of distribution of the data, a 2-tailed Mann Whitney $U$ test was applied; medians with interquartile range, minimum, and maximum values are represented; ${ }^{*} P<0.05$. (E) $N k x 6.1$ and Islet immunostaining at $36 \mathrm{hpf}$ is significantly decreased in the gan line in $84 \%$ of mutants ( $n=21$ of 25 analyzed embryos). (F) Zn8 immunostaining reveals a strong defect in sMN axonal projections at $56 \mathrm{hpf}$ in $72 \%$ of gan ${ }^{\text {del/del }}$ mutants ( $n=18$ of 25 analyzed embryos). Scale bars: $25 \mu \mathrm{m}$ (E); $100 \mu \mathrm{m}$ (F).

To confirm these results and evaluate pure motor capacities, we monitored the spontaneous locomotion of 5 dpf larvae. The spontaneous motility of gan morphants was considerably impaired. While $80 \%$ of the morphants did not move, the remaining moved significantly slower and over a shorter period than control larvae (Figure 1, C and D). The specificity of the motility defects for gigaxonin was demonstrated by the rescue upon coinjection of human GAN mRNA (Supplemental Figure 4B).
Gigaxonin is required for the specification of secondary motor neuron. To explore the role of gigaxonin in sustaining motility in zebrafish, we analyzed the 2 consecutive waves of motor neuron birth in the spinal cord: the primary motor neurons (pMNs) and secondary motor neurons (sMNs), born between 9-16 hpf and 14-51 hpf, respectively (45). Analysis of different key developmental stages using the islet marker revealed a normal proportion of pMNs at $20 \mathrm{hpf}$ (Supplemental Figure 5A), but a substantial reduc- 


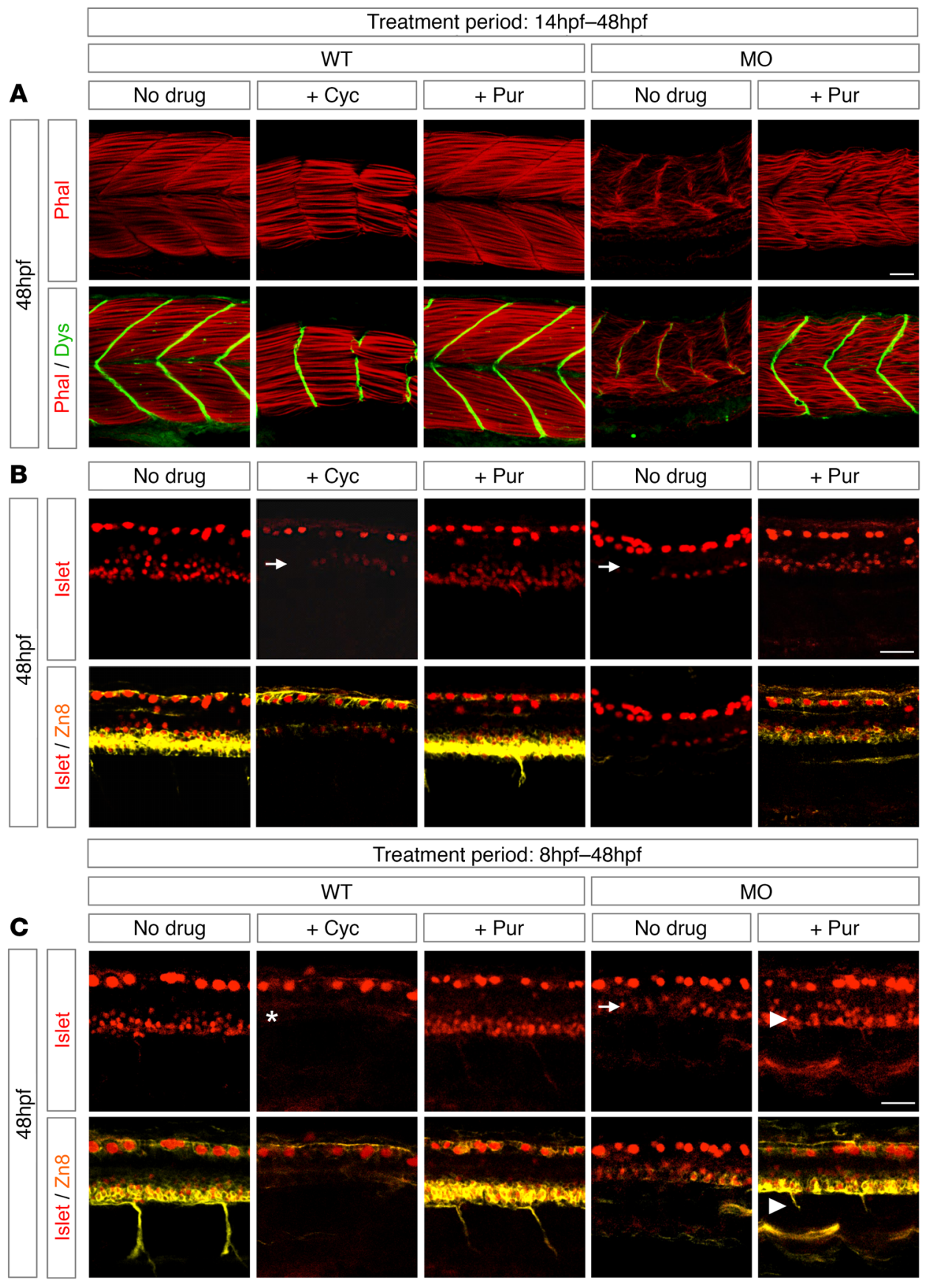

Figure 4. Modulation of Shh activity mimics and restores gigaxonin-dependent phenotypes in zebrafish. ( $A$ and B) Lateral view of WT and MO-injected embryos at $48 \mathrm{hpf}$ after cyclopamine or purmorphamine treatment between 14-48 hpf. (A) MO injection mimics the cyclopamine-induced loss-of-Shh phenotype (U-shape somites [Dystrophin] and less dense myofibers [Phalloidin]), which is partially rescued with purmorphamine. (B) Using the same treatment regime, Islet/Zn8 immunostaining in cyclopamine-treated or $\mathrm{MO}$ embryos reveals a loss of sMNs, while pMNs are still visible (arrows). Purmorphamine treatment partially rescues the loss-of-Shh-like phenotype in gan morphants. (C) Earlier Shh activation by purmorphamine from $8 \mathrm{hpf}$, during the wave of pMN birth (as shown by absence of pMNs in control embryos with cyclopamine: white asterisk), is effective in reversing the defects in SMN specification in the gan morphant (arrowheads). Representative pictures of (A) $n=18 / 18$ (WT), $n=18 / 18$ (WT+Cyc), $n=18 / 18$ (WT+Pur), $n=18 / 18$ (MO), $n=$ $5 / 21$ (MO+Pur); (B) $n=30 / 30$ (WT), $n=$ 35/35 (WT+Cyc), $n=30 / 30$ (WT+Pur), $n=35 / 35$ (MO), $n=7 / 40$ (MO+Pur); and (C) $n=56 / 56$ (WT), $n=49 / 49$ (WT+Cyc), $n=73 / 74$ (WT+Pur), $n=46 / 49$ (MO), $n=$ 53/76 (MO+Pur). Scale bars: $25 \mu \mathrm{m}$.

tion of sMNs at $36 \mathrm{hpf}$ in morphants compared with the control group (Figure 2A). The similarities with zebrafish carrying mutations in the Shh pathway (46-48) prompted us to test whether the decrease in $\mathrm{MN}$ number might result from an impaired cell specification in the gan morphants. For that purpose, we labeled MN progenitors with the Shh target gene Nkx6.1. While the number of Nkx6.1-positive progenitor cells was not notably affected in morphants at $20 \mathrm{hpf}$ (Supplemental Figure 5A), Nkx6.1 expression was markedly reduced prior to sMN differentiation from $28 \mathrm{hpf}$ onward (Supplemental Figure 5, B and C).

These data indicate that gigaxonin depletion inhibits sMN specification through a decrease of Nkx6.1 expression in progenitor cells, as would an inhibition of Shh signaling during the second wave of MN birth. As a result, the structure of the spinal cord was severely impaired in older embryos, with decreased motor neurons as revealed by ultrastructural examination at 72 hpf (Supplemental Figure 6A). We found this effect of gigaxonin to be specific to spinal motor neuron specification, as the Isletpositive branchiomotor neurons in the hindbrain were not affected in gan morphants at $48 \mathrm{hpf}$ (data not shown). Collectively, our data demonstrate a role of gigaxonin in controlling the differentiation of secondary motor neurons in the zebrafish spinal cord.

Gigaxonin controls the axonal pathfinding of primary motor neurons. The absence of sMN in gan morphants was further confirmed by immunostaining using the specific neuronal cell surface marker Zn8. Indeed, Zn8-positive cells were completely absent in morphants 

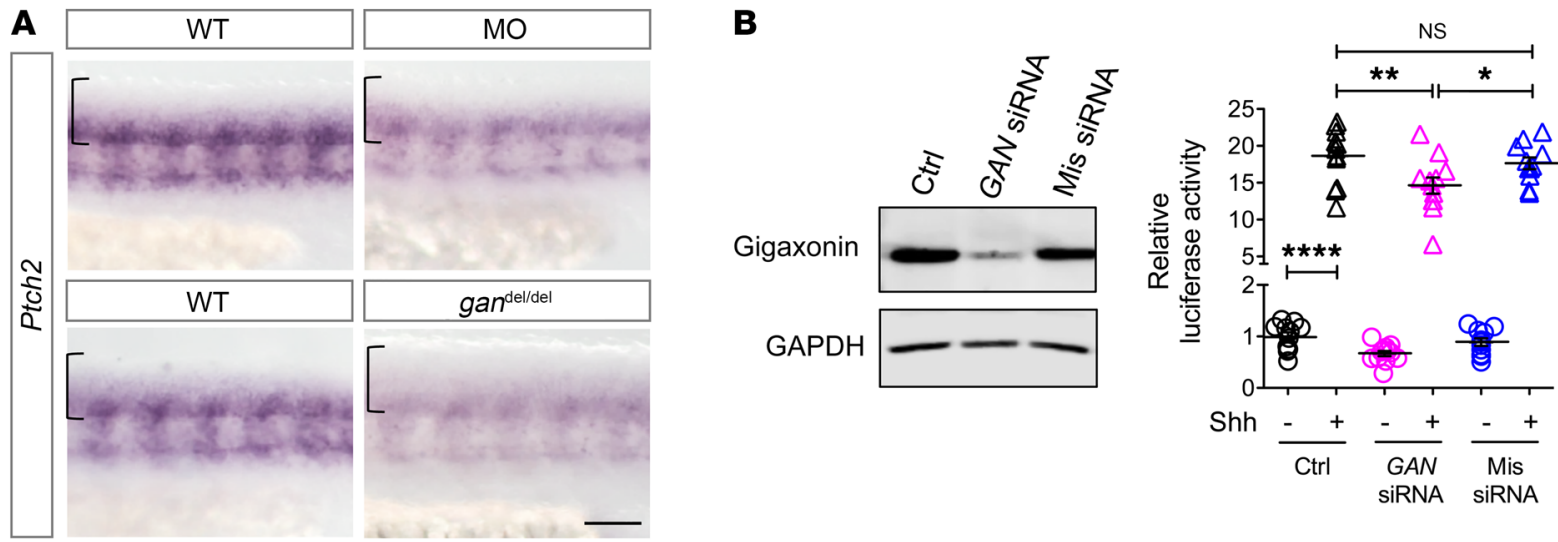

Figure 5. Gigaxonin depletion induces a decrease in Shh activation in zebrafish and in a mammalian reporter cell line. (A) Lateral view of zebrafish spinal cord at $32 \mathrm{hpf}$ showing a reduction of ptch2 mRNA expression in both MO-injected and gan ${ }^{\text {del/del }}$ animals. Spinal cord is indicated by square brackets. (B) Quantification of Shh activation in Shh Light-2 cells depleted in gigaxonin. Transient knock down of $G A N$, as revealed by immunoblotting (left). Quantification of the luciferase activity in Shh Light-2 cells depleted in gigaxonin, with or without treatment with Shh-CM for 48 hours. In the absence of gigaxonin, the Shh-induced luciferase activity is diminished (right). Statistics: with normality of the distribution of the data, a 1-way ANOVA test (with Bonferroni's post hoc test) was used, mean \pm SEM; $n=11-12$ independent experiments, performed in triplicate; NS, not statistically significant; ${ }^{*} P<0.05,{ }^{* *} P<0.01 ;{ }^{* * *} P<0.0001$. Scale bars: $50 \mu \mathrm{m}$.

at $56 \mathrm{hpf}$ (Figure 2B), when sMN axonogenesis in control embryos is finished. Next, we determined whether gigaxonin-depleted pMNs exhibit any defects in axonal pathfinding. At $56 \mathrm{hpf}$, repression of gigaxonin resulted in a wide range of axonal defects. Concomitant with an increased arborization of primary motor axons, gan morphants exhibited an aberrant growth of pMN axons reminiscent of Shh mutants (46), including an absence or significant shortening of the caudal primary (CaP) axons (Figure 2, C-E) and misguided axons with ectopic ventral projections. Interestingly, the 3D view of $48 \mathrm{hpf}$ morphants indicated additional phenotypes (Figure 2D, left panel and Supplemental Videos 2 and 3). Indeed, morphants exhibited abnormal protrusions of axons from the spinal cord, and an apparent absence of the MiP and RoP motor axons, leading to an overall profound alteration of the structure of the spinal tracts and an increased spacing between the 2 motor columns.

Importantly, the axonal deficits and the absence of sMN were reproduced by targeting an independent region of the zebrafish gan mRNA (MO ex1-2) (Supplemental Figure 7A), and rescued upon coinjection of human GAN mRNA (Supplemental Figure $4 C$ ), hence demonstrating the specificity of gigaxonin depletion.

Gigaxonin promotes muscle innervation and somitogenesis. The severity of the defects in both pMN and sMN, together with the locomotion disabilities in morphants prompted us to investigate the integrity of the neuromuscular junction and muscles. Visualization of acetylcholine receptors using $\alpha$-bungarotoxin staining revealed a total absence of synapses along the axons at $48 \mathrm{hpf}$, as compared with control embryos (Figure 2D and Supplemental Videos 2 and 3). We further analyzed the muscle integrity to reveal profound structural abnormalities of muscle trunk somites in gan morphants at $48 \mathrm{hpf}$. Unlike control zebrafish, which have V-shaped somites and well-organized myofibers, gan morphants exhibit U-shaped somites with an absence of horizontal myoseptum, and wavy, less-dense myofibers (Supplemental Figure 6B). Similar to Shh inactivation $(46,48)$, this effect was further reproduced using MO ex1-2 for gan (Supplemental Figure 7B) and was detected as early as $28 \mathrm{hpf}$, concomitant with the abnormalities of pMN axonal pathfinding (data not shown), which may suggest a dual effect of gigaxonin in neuronal and muscle development. Ultrastructural examination of morphant muscles at a later stage further revealed a substantial alteration of myofiber structure, characterized by a marked shrinking of myofibers, an apparent denser content, and an invasion of conjunctive tissue (Supplemental Figure 6C). While control embryos presented a regular hexagonal arrangement of the thick filaments with intercalating thin filaments (Supplemental Figure 6C), morphants exhibited a disorganization of sarcomeres, with a pronounced disparity in the spacing and distribution of the myosin and actin filaments.

Gan deletion zebrafish mutant recapitulates the gan morphant phenotype. To confirm the specificity of our Shh-like phenotypes for gigaxonin, we generated a gan knockout zebrafish line ( $\left.\mathrm{gan}^{\mathrm{del} / \mathrm{del}}\right)$ by deleting the entire endogenous gene (Figure 3A). Interestingly, large mutations have been evidenced in patients with $\operatorname{GAN}(19,49)$. Similar to the gan morphants, we found that $\mathrm{gan}^{\mathrm{del} / \mathrm{del}} \mathrm{zebrafish}$ display normal morphology at early stages of development, but present with severe locomotor impairments. Using a touch-response assay at $72 \mathrm{hpf}$, we identified $49.1 \%$ of gan $^{\text {del/del }}$ zebrafish with swimming defects, among which $76 \%$ of gan $^{\text {del/del }}$ zebrafish were unable to move (Figure 3B). The impairment of motor function was directly confirmed at $5 \mathrm{dpf}$, when $80 \%$ of the total mutants exhibited severe defects in spontaneous locomotion assay, with a significant reduction in both total distance and net velocity (Figure 3, C and D).

We then examined the specification of sMNs by labeling MN progenitors with the transcription factor Nkx6.1. As observed in morphants, the number of Nkx6.1-positive cells was dramatically decreased in $84 \%$ of gan $^{\text {del/del }}$ mutants at 36 hpf (Figure 3E). No significant change was found in the number of $\mathrm{MN}$ progenitor cells at earlier developmental stages, between 20-24 hpf (Supplemental Figure 5).

At $56 \mathrm{hpf}$, gan deletion resulted in a substantial reduction in the number of sMNs and an aberrant growth of their axons, as labeled with the markers Islet and $\mathrm{Zn} 8$, respectively (Figure $3 \mathrm{~F}$ ). This axonal phenotype occurred in $72 \%$ of gan $^{\mathrm{del} / \mathrm{del}}$ zebrafish. 
A
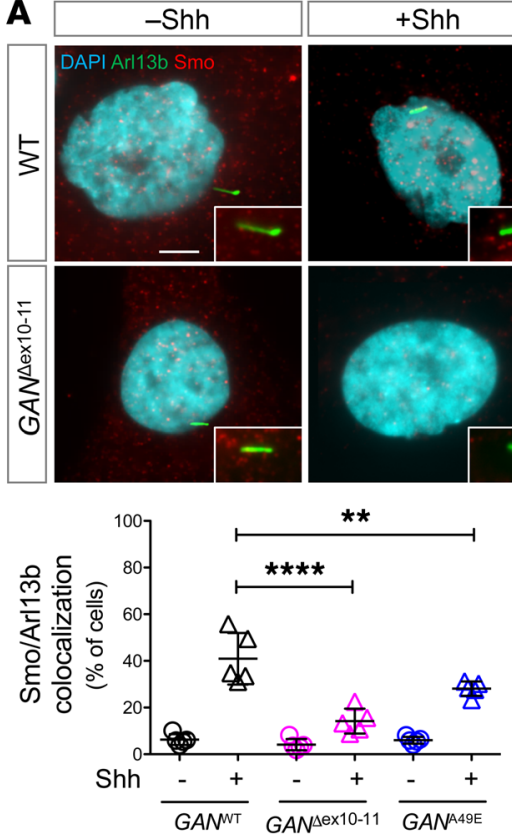

B
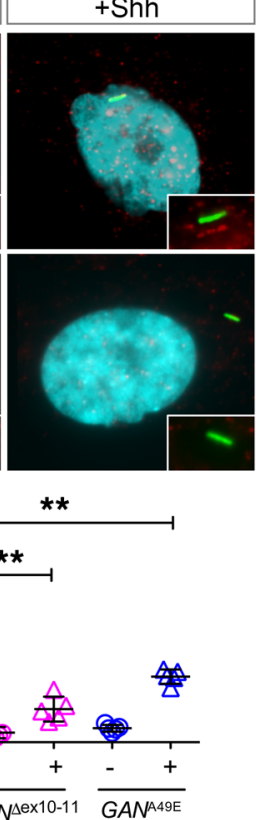
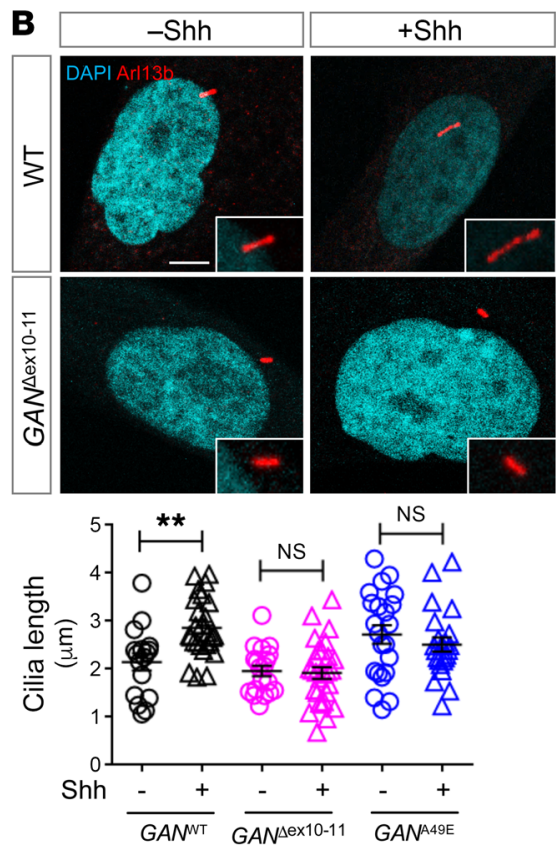

Figure 6. Decreased responsiveness of primary fibroblasts from patients with GAN to the activation of the Shh pathway. (A) Shh-CM treatment for 4 hours causes translocation of Smo into the cilium (arl13b) in control but this is greatly decreased in patient primary fibroblasts, bearing distinct mutations in the GAN gene: GAN ${ }^{\triangle \mathrm{ex} 10-11}$ and $C A N^{A 49 E}$. Quantification of the proportion of Smo-positive cilia in control and GAN fibroblasts, with or without Shh treatment, is shown at the bottom. Statistics: proportions of experimental groups were compared with the $\chi^{2}$ test, data represent mean $\pm \mathrm{SEM} ; n=419\left(\mathrm{GAN}^{\mathrm{WT}}\right), n=$

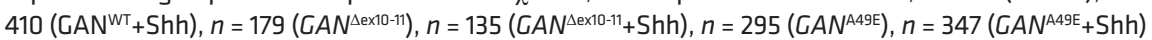
from 5 independent experiments; ${ }^{* *} P<0.01,{ }^{* * *} P<0.0001$ correspond to the comparison between patient and control cells in the presence of Shh (pairwise comparisons of patient with control cells are not statistically significant in the absence of Shh). (B) Shh-CM treatment for 24 hours increases the length of primary cilia in control but not in $C A N^{\Delta \mathrm{ex10-11}}$ and $C A N^{A 49 E}$ patient primary fibroblasts. Quantification of the ciliary length in human fibroblasts, showing a significant increase upon Shh-CM stimulation in control but not in mutant cells, is shown at the bottom. Statistics: with normality of the distribution of the data, a 1-way ANOVA test (with Bonferroni's post hoc test) was used, mean $\pm \mathrm{SEM} ; n=24$ (GAN $\left.{ }^{\mathrm{WT}}\right), n=26$

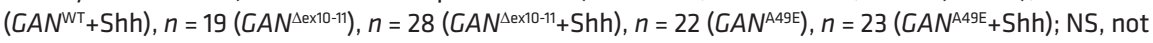
statistically significant; ${ }^{*} P<0.01$. Scale bars: $10 \mu \mathrm{m}$. Insets: Original magnification $\times 2$.

Altogether, the similarity of the phenotypes between the gan genetic mutants and the transiently inactivated z-gigaxonin animals demonstrate that the neurodevelopmental deficits observed in this study are specifically controlled by the gigaxonin encoding gene.

Shh activation restores neuronal specification and somitogenesis deficits in gigaxonin-depleted zebrafish. Shh signaling is crucial for the specification of neuronal identity and for somitogenesis. Genetic and pharmacological ablation of Shh pathway in zebrafish has been shown to abolish motor neuron specification in the spinal cord and to generate U-shaped somites $(42,45,47,48,50)$. The striking similarities with our morphants indicate a potential role of gigaxonin in regulating Shh activity in zebrafish. A first validation toward a downregulation of the Shh pathway in GAN was provided by the pronounced reduction in the expression of the Shh responsive target Nkx6.1 gene in the spinal cord of gan morphants (Figure 2A). To further expand on these results, we modulated Shh signaling in zebrafish, to either inhibit or activate it, using cyclopamine and purmorphamine, respectively. We demonstrated that cyclopamine administration during the second wave of MN birth (from $14 \mathrm{hpf}$ to $48 \mathrm{hpf}$ ) in WT embryos fully reproduces the phenotype induced by sion was ablated. First, we performed in situ hybridization on the gan morphants and the $g a n^{\text {del/del }}$ line to assess the expression pattern of ptch2, a direct target of Shh signal transduction and a well-established indicator of Shh activation (Figure 5A). In the spinal cords of zebrafish embryos at $32 \mathrm{hpf}$, ptch 2 displayed a ventral-high, dorsal-low pattern of expression. In conditions where gigaxonin expression was reduced, either through MO knockdown or genetic ablation, the expression of ptch 2 in the spinal cord was dramatically decreased (Figure 5A), hence evidencing an inhibition of Shh signaling in the absence of gigaxonin. Second, we knocked down gigaxonin with RNAi in Shh Light-2 cells (Figure 5B, left panel), an NIH-3T3 cell line that is stably expressing a Gli-dependent luciferase reporter and becomes activated upon Shh stimulation (51). In this study, we demonstrated that while Shh alone increased the activity of the luciferase 19-fold, the decrease in gigaxonin levels diminished this effect by 22\% (Figure 5B, right panel). Importantly, this effect was only substantial in the presence of the morphogen, and not under basal conditions, which indicates an effect specifically upon Shh activation. Third, we analyzed primary fibroblasts derived from GAN patients, 
A

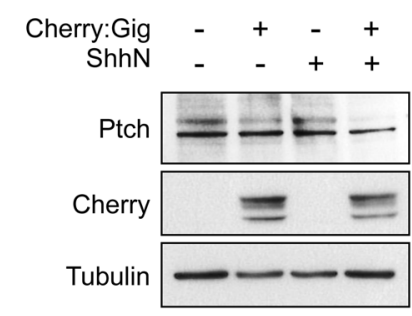

C

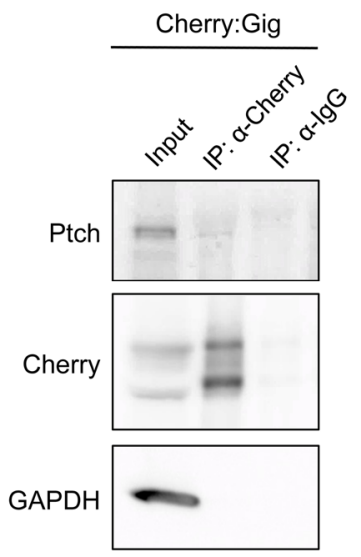

B

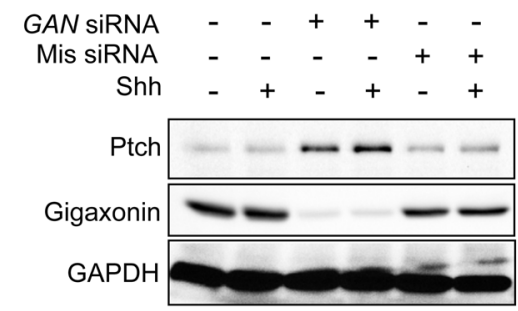

D

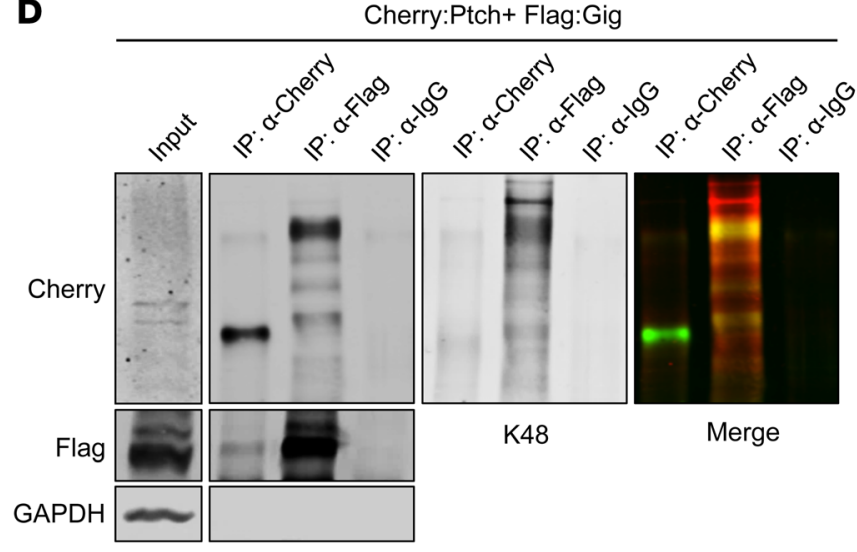

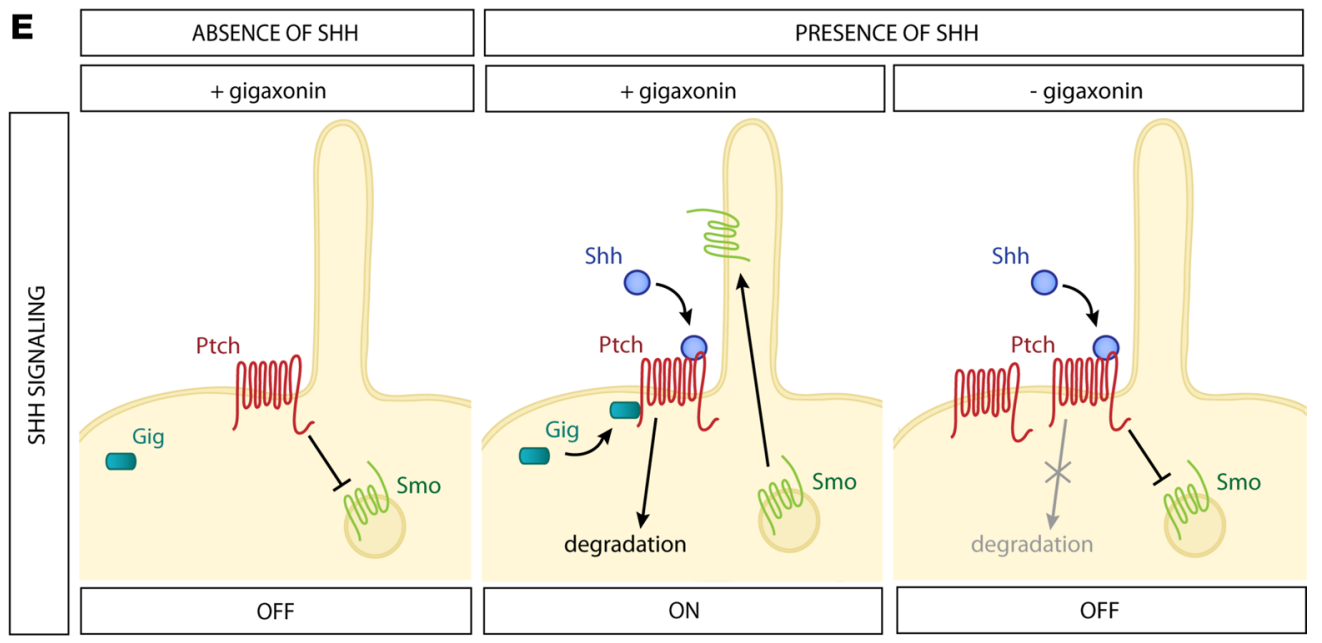

Figure 7. Shh-dependent control of Ptch abundance by gigaxonin. (A) $\mathrm{NIH}-3 \mathrm{~T} 3$ cells were transfected with human Cherry-Cig plasmid $\pm 3 \mu \mathrm{g}$ / $\mathrm{mL}$ Shh for 48 hours. Degradation of endogenous Ptch protein occurs only in the presence of both ectopic gigaxonin and Shh. (B) Repression of the endogenous gigaxonin using siRNA causes an increase of the endogenous Ptch levels in Shh Light-2 cells, which is potentiated by Shh induction. (C and D) Interaction between gigaxonin and Ptch, as revealed by reverse immunoprecipitation on both endogenous (C) and ectopic (D) Ptch protein. Shh Light-2 cells transfected with human Cherry-Gig (C); COS-7 cells transfected with zebrafish 3Flag-Gig and zebrafish Cherry-Ptch (D). IgG serves as an internal negative control. (D) Cherry immunoprecipitation identifies Ptch proteins, which are mainly not modified, while the Ptch pool enriched in gigaxonin immunocomplex presents a laddering overlapping with K48-specific ubiquitin chain. (E) Model of action of the gigaxonin-E3 ligase in the initiation of Shh signaling. In an off state (left), prior to Shh activation, receiving cells silence the cascade through the inhibitory effect of the Ptch receptor on the effector Smo. Upon Shh production, the active Shh form is released and received by progenitor cells. Gigaxonin acts as an initiator of signal transduction by degrading Shh-bound Ptch receptor (middle), hence allowing the derepression of the signal transducer Smo, which translocates into the cilium to activate the pathway. In the absence of gigaxonin (right), receiving tissues are unable to interpret Shh signaling, due to the constitutive repression of Smo induced by the accumulation of Shh-bound Ptch receptor. which represent a well-established cellular model for the human pathology $(25,26)$. We selected independent primary fibroblasts carrying different mutation types representative of the pathology: a large deletion $\left(G A N^{\Delta \mathrm{ex10-11}}\right)$ and a missense mutation $\left(G A N^{\mathrm{A} 49 \mathrm{E}}\right)$ for which gigaxonin was dramatically reduced (19). Conveniently, human primary fibroblasts present primary cilium, the antenna for Shh signaling in vertebrates, which can be easily detected. An established and well-documented method to identify Shh activation is the detection of the translocation of Smo into the cilium in the presence of the morphogen (52). Here we showed that in control human fibroblasts exposed to Shh, Smo localization to the cilium was significantly increased, whereas this action was dramatically reduced in GAN patient fibroblasts (Figure 6A). Not only does the Shh pathway rely on the presence of cilium for signal transduction, but intraflagellar transport particles of the cilium and downstream targets of Shh have also been shown to regulate cilium length to modulate Shh signaling (35). Thus, as a second readout for Shh responsiveness, we compared the cilium length between control and mutant primary fibroblasts upon Shh induction. Interestingly, while the control human fibroblasts exposed to Shh increased the cilia length by $29 \%$ (Figure 6B), the ciliary length of the independent GAN fibroblasts was not altered upon Shh addition, hence showing their inability to respond to Shh activation. Altogether, we demonstrate here that Shh signaling fails to activate properly in the absence of gigaxonin in zebrafish, mouse, and human systems, hence providing substantial evidence for the critical role of gigaxonin in promoting Shh induction.

Gigaxonin acts together with Shh to degrade the Ptch receptor and initiate the signaling cascade. To gain insight into the molecular mechanism by which the gigaxonin-E3 ligase positively controls Shh signaling, we directly addressed whether gigaxonin can act together with Shh to target Ptch for degradation, and therefore 
initiate the Shh pathway. Using both loss- and gain-of-function methodologies, we investigated the levels of endogenous Ptch in the presence or absence of Shh. Overexpressing gigaxonin, with or without Shh, provided evidence that endogenous Ptch could only be efficiently degraded when both gigaxonin and Shh were added to the system (Figure 7A). Conversely, reduction of the levels of endogenous gigaxonin with RNA interference revealed an increase in the abundance of endogenous Ptch, which was exacerbated when Shh was present (Figure 7B). From these results, we hypothesized that gigaxonin might interact with Ptch. Indeed, using a coimmunoprecipitation (co-IP) assay in Light- 2 cells, we detected the presence of endogenous Ptch in the gigaxonin complex (Figure 7C). We confirmed these results by overexpressing both Cherry-Ptch and Flag-Gig in COS-7 cells and performing co-IP in both directions (Figure 7D). Interestingly, we observed multiple bands with Cherry-Ptch in gigaxonin immunocomplex, resembling the laddering characteristic of ubiquitination. We confirmed this by colabeling the Cherry-Ptch signal with anti-ubiquitin antibody exhibiting specificity for lysine-48 linkage, hence identifying poly-ubiquitinated Ptch in gigaxonin complex. The comparison with Ptch immunocomplex was also informative. Beyond the confirmation of the interaction of both proteins, the absence of laddering of Ptch and the weaker pull down of gigaxonin revealed that while gigaxonin does not interact with the entire pool of Ptch within the cell, the gigaxonin complex is significantly enriched in ubiquitinated Ptch. Collectively, our data demonstrate that gigaxonin acts positively on the Shh pathway, through the interaction with the Ptch receptor and a Shh-dependent targeting for degradation.

\section{Discussion}

In this study, we combined physiological evidence, cellular assays, and biochemical data to discover a causal role of developmental dysfunctions in the pathogenesis of GAN in the motor system, whereby gigaxonin-E3 ligase loss-of-function inhibits the Shh pathway. Controlling the degradation of the Ptch receptor, the upstream component of the cascade, and in a Shh-dependent manner, we propose gigaxonin-E3 ligase as a key molecular switch for the initiation of the signal transduction mediated by Shh (Figure 7E). Examined at an organism level in zebrafish, this gigaxonin-mediated regulatory mechanism is essential for specifying both neuronal and muscle patterning to sustain locomotor activity in vivo, and its perturbation mirrors the loss of ambulation seen in GAN patients. Notably, the ability of the human gigaxonin to fully rescue the deficits in the gan zebrafish model, combined with the evidence of an impairment of Shh signaling in patient cells, support a functional conservation of this developmental signature in patients.

Here, we provide conclusive evidence of the physiological role of gigaxonin in positively regulating the Shh pathway in vivo. First, we show that gigaxonin depletion mimics the loss-of-Shh phenotype. Both MN specification and somitogenesis are compromised in gan morphants, causing sMN loss and U-shape somites similar to the pharmacological and genetic inhibition of the Shh pathway through loss-of-function of Smo $(46,48)$, Gli $(47,50)$, or Shh $(53)$. Second, we show that the absence of sMN results from the impairment in the differentiation of progenitor cells, as evidenced by the downregulation of the Shh-responsive Nkx6.1 target gene from $28 \mathrm{hpf}$ onwards. These Shh-like phenotypes and markers were also evidenced in the CRISPR gan knockout zebrafish line, which demonstrates the specificity of the phenotypes toward gigaxonin functions. Third, in situ hybridization in both gan morphants and the $g a n^{\text {del/del }}$ line revealed a reduced expression of $p t c h 2$, a target of Shh cascade, hence directly demonstrating the inactivation of Shh signaling in zebrafish depleted in gigaxonin. Last, pharmacological activation of the Shh pathway was able to restore both neuronal specification and somitogenesis in gan morphants, in agreement with the function of gigaxonin in positively regulating Shh activity. We further confirmed this statement in independent biological assays, using both a mouse Shh reporter cell line depleted for gigaxonin and human primary fibroblasts derived from GAN patients. Using established readouts for Shh activation, i.e., transcriptional activation (51), Smo localization to cilium (52), and cilium length (35), we demonstrated in both cellular systems an impairment of gigaxonin-depleted cells to respond to Shh induction.

Among other morphogenic pathways (Wnt, BMP, FGF, etc.), Hedgehog is unique as it relies on a paucity of activating ligands and on a single downstream signaling cascade, hence placing gigaxonin as a key molecular determinant to decipher its physiological regulation, necessary to ensure spatio-temporal and process specificity. In this study, we add insights into the regulation of the Shh pathway in the context of the motor system. Our investigation of $\mathrm{MN}$ birth, which occurs in 2 sequential waves in zebrafish (9-16 hpf for pMNs and 14-51 hpf for sMNs) (45), revealed a specific impairment of sMN specification during the second wave in gan morphants. In these animals, pMN specification seemed correctly achieved, but severe defects were observed during the axogenesis process, which takes place during the first day of development. While the maternal gan mRNA (as shown in Supplemental Figure 2A) may partially compensate for Shh activation during the first wave of MN birth, our results may also suggest a differential and temporal regulation of the canonical and noncanonical roles of Shh by gigaxonin. Indeed, the misguidance of CaP pMN axons in gan morphants, which are comparable to Smo mutants (46), may be caused by the reported noncanonical functions of Shh in controlling axon guidance and axon fasciculation $(54,55)$. Intriguingly, our pharmacological experiment using purmorphamine revealed that the restoration of sMN specification was not efficient when applied during sMN birth, but required a treatment during the first wave. These data reinforce the notion that the 2 critical periods of $\mathrm{MN}$ induction are not independent and that Shh activity in the first wave is a key temporal determinant for the second one, as previously illustrated by the absence of sMN in embryos subjected to cyclopamine treatment for a short 4-10 hpf period (46). Thus, our results reveal severe defects in $\mathrm{pMN}$ axonal pathfinding in morphants and may offer interesting speculations on the control of $\mathrm{MN}$ fate by axonal determinant.

While this study provides important insight into the control of the Shh pathway by gigaxonin within the motor system, it might also be possible to extend this insight to other modalities, within and outside the nervous system. Indeed, we showed that gigaxonin is enriched in the nervous system and during prenatal stages (17), but its expression is ubiquitous. Thus, one could suggest that the coexistence of Shh cascade and gigaxonin within the same tissue would provide the context specificity of this control, extending beyond the nervous system. Accordingly, Shh signaling has been implicated in the development of multiple nonneuronal tissues 
Table 1. List of primers

\begin{tabular}{|c|c|c|c|}
\hline & & & Primers \\
\hline PCR & gan exons 3-4 & Forward & 5'-AGAAGCTCAACGTTGGGAA-3' \\
\hline \multirow[t]{3}{*}{ In situ hybridization } & gan antisense probe & Forward ${ }^{A}$ & 5'-AATTAACCCTCACTAAAGGGACAgaaactcacggacatagtcgaa-3' \\
\hline & & Reverse & $5^{\prime}$-attgttgtatggagtctct-3' \\
\hline & gan sense probe & Forward ${ }^{A}$ & 5'-AATTAACCCTCACTAAAGGCAGAattgttgtatggagtctcta-3' \\
\hline \multirow[t]{3}{*}{ Genotyping } & gan exon 1 & Forward & 5'-CAGCACATCTGTCTGTACTGTG-3' \\
\hline & & Reverse & 5'-CAGCAGCTACTCATCTCATCTC-3' \\
\hline & gan exon 11 & Reverse & 5'-GCATCAAGATACTAGCCGTATC-3' \\
\hline \multirow[t]{3}{*}{ Morpholino oligonucleotides } & gan & Mo ex2-3 & 5'-AGAGTGATCTACAGAAGGAAACAGT-3' \\
\hline & gan & Mo ex1-2 & 5'-TTGGTCCTGGAAACACAGTAACACA-3' \\
\hline & gan & Mis ex2-3 & 5'-AGAGTcATgTACAcAAGcAAACAcT-3' \\
\hline & & Reverse & 5'-ggggaccactttgtacaagaaagctgggttGAGCCGTATTCTCAGTCTGT-3' \\
\hline \multirow[t]{2}{*}{ Cene inactivation } & GAN & SiRNA & 5'-P-UAUCCCUUCAAGUUCAAUCUU--3' \\
\hline & Mis & siRNA & 5'-UAUCaCUUCAAGUcCAAUCUU-3' \\
\hline
\end{tabular}

AUppercase: T3 promoter sequence. ${ }^{B}$ Lowercase: ATTB sequence.

(29), including skin, hair, mammary gland, stomach, and kidney, all of which are reported to be affected in GAN patients (13). As the substrate adaptor of an E3 ligase complex, the ubiquitous gigaxonin may also be functionally silenced in tissues insensitive to Shh, through posttranslational modifications, or by limited amount of the other subunits. Thus, GAN is commonly defined as a neurodegenerative disease, due to the massive deterioration of the entire nervous system, but should be more adequately investigated beyond the nervous system to shed light into other possible damaging phenotypes that would reflect a global impairment of Shh signaling, as extrapolated from our study.

E3 ligases and ubiquitination constitute a pivotal mechanism of Shh regulation, by providing both negative and positive feedback loops on Shh activation. Therefore, they play a key role in fine-tuning Shh responses. Although regulation is well studied for the transcription factor $\mathrm{Ci} / \mathrm{Gli}$, little is known about the regulation of the early steps of Shh activation. Previous studies have shown that ubiquitination of the transducer Smo and the Shhbound Ptch receptor are essential to trigger Shh activation, but the identity of the E3 ligase(s) that control(s) the initiation of the pathway is unknown. Here, we show that the gigaxonin-E3 ligase plays a role in the early steps of the Shh cascade, mediating the degradation of the Ptch receptor necessary to relieve the constitutive inhibition of Smo. We showed that gigaxonin interacts with endogenous Ptch and that its degradation is potentiated by the presence of Shh, hence placing gigaxonin as the first E3 ligase, to our knowledge, that enables the receiving cells to interpret the morphogen signal. Indeed, the Itchy and Smurf E3 ligases act on Ptch to control the basal turnover of the receptor in the absence of the morphogen (44), or unbound Ptch as a mechanism to turn off Shh signaling, respectively (42).
We previously identified the gigaxonin-E3 ligase (15) and 2 of its targets: the IF proteins $(20,24)$ and the autophagy protein ATG16L1 (28). Through direct binding and ubiquitination, gigaxonin controls the steady state of these cellular compounds to balance the homeostasis of the cytoskeleton within neuronal and nonneuronal cells and regulate the autophagic flux within neurons. These studies have uncovered the crucial role of gigaxonin in fine-tuning 2 fundamental cellular processes, but the demonstration of their respective implication in neuronal dysfunctions in GAN has yet to be provided. The characterization of the neurodegenerative model in GAN neurons (28) will permit such investigation in the future. While we do not exclude any potential contribution of the known effectors of gigaxonin to disease, we have identified here the Shh pathway as a novel target for gigaxonin, which is sufficient to reproduce the motor deficits seen in patients.

To closely mimic the genetic spectrum of mutations in GAN, we deprived the zebrafish of gigaxonin, using a transient and a stable knockout approach. Both systems revealed severe and penetrant motor deficits, with loss of locomotion in $80 \%$ of z-gigaxonin-depleted animals, as a result of denervation, impaired motor neuron specification, and somitogenesis, hence supporting a loss-of-function mechanism in GAN. To our knowledge, the zebrafish model we describe here represents the first and heretofore most robust animal model for GAN, given that it emulates the severity and penetrance of the motor deficits seen in patients $(13,14)$. Indeed, attempts by our and other laboratories revealed only modest and late-onset sensory-motor symptoms in distinct GAN mice, with no overt neuronal damage $(17,22)$. Future examination of the phenotype and survival of the adult-stage zebrafish model is important. In particular, it could confirm locomotion defects in adult animals, and permit investigation of the myriad 
of late-onset symptoms that patients develop in the CNS, including ataxia, dysarthria, nystagmus, vision impairment, intellectual disabilities, and epilepsy.

Noticeably, Shh impairment leads to a pleiotropic phenotype that sums up the variety of symptoms found in GAN patients, in the CNS and PNS. Indeed, mutations in components of the Shh pathway in human cause ataxia (56), vision impairment (57), intellectual disabilities (58) and epilepsy (59). While this has to be confirmed in the gan zebrafish, the similarities of symptoms among the Shh models, GAN patients, and our study support a main role of Shh dysfunction as a causal mechanism in the GAN pathogenesis. This hypothesis is further sustained by the massive atrophy of neuronal tissues in brain MRI of patients, the detection of a morphological marker of developmental deficit in patients (16), and the enrichment of gigaxonin in neuronal tissues and in prenatal stage (17). Thus, further work would be important to determine whether the impairment of Shh signaling during development is sufficient to cause adult symptoms. Awaiting for this demonstration, GAN exemplifies the emerging concept whereby neurodevelopmental deficits disrupt homeostasis and generate vulnerability that further evolves toward clinical manifestations in adulthood, as shown in HD (9-11) and $\mathrm{AD}(7)$ diseases.

To our knowledge, this study provides the first hints for the hypothesis that the human GAN pathology has a neurodevelopmental component, but it also opens an exciting new avenue for a role of the Shh pathway in adulthood. Indeed, in the mammalian adult brain, Shh signaling has emerged as an important neuromodulator through different mechanisms, including the proliferation of postnatal neural stem cells and fate specification $(60,61)$. One could therefore speculate for the human pathology, that the gigaxonin-mediated $\mathrm{Sh}$ repression can act at multiple levels in prenatal and postnatal stages. Since GAN is an early-onset pathology, a more comprehensive study aimed at deciphering the control of Shh signaling by gigaxonin during development and in adulthood could shed light on possible treatment windows for this yet incurable and fatal disease. In light of the therapeutic benefits obtained by modulating the Shh pathway in several neurodegenerative conditions (60), our study may also offer a specific target for therapeutic intervention aimed at reactivating neurogenesis in disease.

\section{Methods}

Genebank zebrafish gan cDNA accession number. The full zebrafish gan cDNA was sequenced and released in the Genebank database under accession number KT013299; the corresponding gigaxonin protein ID is ANJ65950.

Animals and morpholino knockdown. Zebrafish (Danio rerio, Oregon $\mathrm{AB}$ ) were maintained at $28.5^{\circ} \mathrm{C}$ and on a $14 / 10$ hours light/dark cycle and staged by hours or days postfertilization. The gan antisense morpholino oligonucleotides (MOs, Gene Tools) and mismatch morpholino (Mis) were microinjected into 1- to 2-cell-stage embryos according to standard protocols. A volume of $1 \mathrm{~nL}$ was injected at a concentration of $0.25 \mathrm{mM}$ for the MO/Mis ex2-3 and 0.6 mM for the MO ex1-2 (sequences in Table 1).

Reverse transcription PCR (RT-PCR). mRNA extraction was performed on dechorionated embryos at specific times using Trizol (SigmaAldrich). cDNA was obtained after reverse transcription (SuperScript III
Kit, Invitrogen) and gan coding sequences were obtained with the primers gan exons 2-3 and gan exons 3-4, as presented in Table 1.

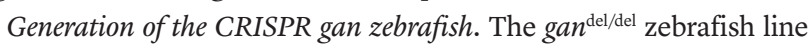
was generated using the genome-editing technology CRISPR (AMAGEN). Six distinct guide RNAs were selected against off-target genes, using the CRISPOR tool (http://crispor.org; ref. 62) and designed within exon 1 and exon 11 of the zebrafish gan gene. They were coinjected with Cas9 protein into zebrafish eggs, and their efficacy and toxicity were compared at $48 \mathrm{hpf}$. The selected sgRNAs were used to create the $\operatorname{gan}^{\mathrm{del} /+}$ line, whose deletion lies between the middle of exon 1 and downstream of the stop codon in exon 11 . As confirmed by sequencing, the resulting Open Reading Frame is restricted in exon 1 and contains several premature STOP codons, with only 43 amino acids produced. F3 gan zebrafish were crossed to obtain fertilized gan $^{\text {del/del }}$ eggs, with an average of $10 \%$ lethality. Genotyping was performed as follows: genomic DNA extraction was performed by collecting tail pieces from anesthetized embryos into lysis buffer (10 mM Tris- $\mathrm{HCl}$ pH 8, 2 mM EDTA $\mathrm{pH} 8,0.2 \%$ Triton $\mathrm{X}-100$, proteinase $\mathrm{K}$ ) boiled for 10 minutes at $96^{\circ} \mathrm{C}$. Genomic DNA containing deleted sites was PCR-amplified using primer sequences presented in Table 1 and separated on a $2 \%$ agarose gel.

(Immuno) histochemistry and electron microscopy of zebrafish embryos. Zebrafish were treated with $75 \mu \mathrm{M}$ PTU (1-phenyl 2-thiourea) from 10 hpf to prevent pigmentation. At appropriate developmental stages, they were anesthetized with $0.0168 \%$ tricaine (MS-222, E1052, SigmaAldrich), and fixed in 4\% PFA for 4 hours at room temperature or overnight at $4^{\circ} \mathrm{C}$, and permeabilized in $1 \mathrm{X}$ PBS- $1 \%$ Triton X-100 for 2 hours on an orbital shaker. The embryos were incubated in blocking buffer $(1 \%$ DMSO, $1 \%$ normal donkey serum, 1\% BSA, 0.7\% Triton X-100, PBS) for 1 hour at room temperature and incubated in primary antibodies overnight at $4^{\circ} \mathrm{C}$. Primary antibodies were from following sources: mouse IgG2b anti-islet 1/2 (1:100, 39.4D5, Developmental Studies Hybridoma Bank [DSHB]), mouse IgG2a anti-synaptotagmin 2 (1:100, Znp-1, DSHB), mouse IgG1 anti-neurolin (1:100, Zn8, DSHB), mouse IgG1 antiNkx6.1 (1:20, F55A10, DSHB), mouse IgG1 anti-dystrophin (1:20, MANDRA1 7A10, DSHB), rabbit anti-smoothened (1:150, Ab38686, Abcam), mouse IgG2a anti-Arl13b (1:150, N295B/66, NeuroMab), anti- $\alpha$-bungarotoxin Alexa Fluor 555 conjugate (1:50, B35451, Invitrogen). Following $0.1 \%$ Triton X-100/PBS washes, the embryos were incubated in secondary antibodies overnight at $4^{\circ} \mathrm{C}$. For immunofluorescence, Alexa Fluor 488-, Alexa Fluor 594-, and Alexa Fluor 647-conjugated secondary antibodies were from Jackson ImmunoResearch Laboratory (1:500; catalog numbers 200-542-211, 200-582-211, and 200-602-211, respectively). Imaging was performed using confocal laser scanning microscope model LSM700 (Carl Zeiss). Quantification of projection lengths was obtained with ImageJ software and 3D acquisitions were performed with LightSheet Z.1 microscope (Carl Zeiss). For H\&E contrast, the embryos were fixed in $4 \% \mathrm{PFA} / \mathrm{PBS}$ for 2 hours at room temperature followed by permeabilization in PBS-1\% Triton X-100 for 2 hours. After washes in ethanol, the embryos were incubated for 2 minutes in $0.1 \%$ eosin, washed in ethanol, and incubated in 1:10 hematoxylin for 40 seconds. Bright field images were taken on a SPOT camera mounted on a Zeiss Axioplan 2 imaging microscope. TEM analysis has been performed in 72 hpf embryos, using protocol previously described (63).

Locomotion assays. The touch-response test was performed on 72 hpf embryos, by a slight mechanical stimulation. The motion of individual larva was examined and scored as normal swimming, looping swimming, pinwheel swimming, or motionless. Representative track- 
ing from movies was obtained with ImageJ software. The spontaneous motility of zebrafish was monitored at $5 \mathrm{dpf}$, using the Zebrabox recording system (Viewpoint). The tracking of the motility was recorded for individual zebrafish on a 96-well plate for 1 hour, and presented as slow (3-6 mm/s) and high velocities ( $>6 \mathrm{~mm} / \mathrm{s})$.

Pharmacological modulation of Shh pathway in zebrafish. In vivo inhibition and activation of Shh signaling was achieved with incubation of dechorionated embryos in $50 \mu \mathrm{M}$ cyclopamine hydrate (C4116, SigmaAldrich) and purmorphamine (SML0868, Sigma-Aldrich), respectively. Analysis of embryos was performed at $48 \mathrm{hpf}$, after a treatment window targeting the birth of both primary and secondary motor neurons (from $8 \mathrm{hpf}$ ) or uniquely the secondary wave (from $14 \mathrm{hpf}$ ).

Cloning of early players of the Sonic hedgehog pathway in zebrafish. Zebrafish mRNAs were extracted using Trizol (Sigma-Aldrich) and subjected to reverse transcription (SuperScript III Kit, Invitrogen). cDNAs of Ptch1 and gigaxonin were amplified by PCR using primers flanked with ATTB1/ATTB2 sequences and cloned in the pcDNA-mCherry-N and $\mathrm{pCi}-3 \mathrm{xFlag}-\mathrm{N}$ gateways vectors, respectively. Gigaxonin cDNA was cloned from plasmid previously described (25) in the pcDNA-mCherry-N gateway vector. See Table 1 for primer sequences.

Cell culture. NIH-3T3 cells (ATTC, clone CRL-1658) were grown in DMEM supplemented with $10 \%$ new bovine calf serum, $1 \%$ penicillin, $1 \%$ streptomycin. Light-2 cells (provided by P. Beachy, GRCF Biorepository \& Cell Center, Johns Hopkins University, Baltimore, Maryland, USA) were cultured in DMEM supplemented with $10 \%$ FBS, $0.4 \mathrm{mg} /$

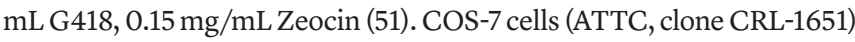
were grown in DMEM supplemented with $10 \%$ FBS, 1\% penicillin, 1\% streptomycin. Transfection with pci 3xFlag-Gig, pcDNA-mCherry-Ptch/ Gig plasmids was performed using Lipofectamine 2000 (Thermo Fisher Scientific). At 24 hours after transfection, NIH-3T3 cells were treated with $3 \mu \mathrm{g} / \mathrm{mL}$ ShhN (1314-SH, R\&D Systems) (39) and Light-2 cells with Shh-conditioned medium (Shh-CM) obtained from ShhN-expressing HEK293 cells for 48 hours (provided by P. Beachy, GRCF Biorepository \& Cell Center, Johns Hopkins University, Baltimore, Maryland, USA) (64). GAN and mismatch siRNA were transfected using DharmaFECT 1 Transfection Reagent (see Table 1 for sequences). Human control and GAN patient fibroblasts carrying different homozygous mutation types, including large deletion ( $\left.G A N^{\mathrm{Aex10}-11}\right)$ and a missense mutation $\left(G A N^{\mathrm{A49E}}\right)$ $(19,25,26)$, were grown in DMEM supplemented with $10 \%$ FBS, $1 \%$ penicillin, $1 \%$ streptomycin. For the ciliary staining, cells were plated on glass coverslips overnight, serum starved in 0.5\% FBS for 24 hours, and supplemented with Shh-CM for 4 or 24 hours, as indicated.

Biochemistry. At 48 or 72 hours after transfection, lysis of cells and immunoblotting were performed as previously described (26). Briefly, the cells were incubated in lysis buffer (50 mM Tris pH 7.5, $150 \mathrm{mM} \mathrm{NaCl}, 1 \%$ Triton X-100, $5 \mathrm{mM}$ EDTA, and a cocktail of protease inhibitors) for 30 minutes and the supernatant retrieved by centrifugation. The proteins were separated on $8 \%$ or $10 \%$ SDS polyacrylamide gel, transferred onto nitrocellulose membranes, and blocked for 1 hour at room temperature in blocking buffer (5\% milk, $0.05 \%$ Tween 20, PBS). Primary antibody incubation was done overnight at $4^{\circ} \mathrm{C}$, followed by PBST $(0.05 \%$ Tween 20, PBS) washes and secondary antibody incubation for 1 hour at room temperature. For the immunoblotting, primary antibodies were from the following sources: mouse anti-GAPDH (1:2000, AM4300, Ambion), mouse anti-tubulin alpha (DM1 $\alpha)$ (1:1000, CP06, Calbiochem), mouse anti-Flag (M2) (1:2000, F3165, Sigma-Aldrich), mouse anti-mCherry
(1C51) (1:2000, Ab125096, Abcam), rabbit anti-Patched1 (1:100, Ab53715, Abcam), rabbit anti-ubiquitin, and Lys48-specific (K48) (1:1000, 05-1307, Millipore). HRP secondary antibodies were from the following sources: goat anti-rabbit (1:5000, 31460, Millipore), goat anti-mouse (1:5000, 31430, Millipore), and rat anti-mouse IgG (1:1000, ab131368, Abcam). Fluorescence-labeled secondary antibodies used for immunoblotting were as follows: donkey anti-mouse IRDye 800 CW (1:15,000, catalog 926-32212, Eurobio), donkey anti-rabbit IRDye 680 RD (1:15,000, catalog 926-68073, Eurobio). Fluorescent and HRP secondary antibodies were revealed using Odyssey Clx (LI-COR) and ChemiDoc XRS+ (BIO-RAD) imagers, respectively. In the co-IP assay, cells were treated with MG-132 12 hours prior to cell lysis, and the immunocomplexes were recovered with Dynabeads Protein G (Invitrogen) linked to specific or control mouse IgG (Santa Cruz, Sc2025) antibodies, after incubation with the cell lysates. For immunofluorescence staining, the cells were plated on 12-mm circular coverslips and fixed in 4\% PFA for $20 \mathrm{~min}$ at room temperature. Cells were blocked for 1 hour at room temperature in blocking solution (4\% BSA, $4 \%$ donkey serum, 0.1\% Triton-X 100, PBS) and stained overnight at $4^{\circ} \mathrm{C}$ in primary antibodies. After washes in PBS, the cells were stained with appropriate secondary antibodies for 1 hour at room temperature, followed by staining with DAPI for 5 minutes (25). Fluorescent images were taken with a confocal laser scanning microscope model LSM700 (Carl Zeiss).

Dual luciferase reporter assay. The dual luciferase reporter assay was performed as previously described (64). Briefly, Shh Light-2 cells were seeded into 24 -well plates the night before transfection. The cells were transfected with GAN or mismatch siRNA (Dharmacon) using DharmaFECT 1 transfection reagent (Dharmacon). At 24 hours after transfection, the cells were switched to low-serum (2\% FBS) medium and treated with conditioned medium from ShN-expressing cells as indicated. At 72 hours after transfection, the cells were lysed and luciferase activity was analyzed on CLARIOstar (BMG Labtech) using the Dual-Luciferase Reporter Assay System (Promega).

Statistics. The statistical significance of the differences between experimental groups was determined by the GraphPad Prism software, except for the comparison of proportions, which was realized by the R software. The assessment of the normality of the distribution of the data was determined with the Shapiro-Wilk test, to apply either a parametric or nonparametric test. Accordingly, the parametric 1-way ANOVA test (with the Bonferroni's post hoc test) was applied for Figure 2E, Figure 5B, Figure 6B, Supplemental Figure 3 (lower panels); the nonparametric Mann Whitney $U$ test was applied for Figure 3D; the nonparametric Kruskal-Wallis test (with the Dunn's post hoc test) was applied for Figure 1D, Supplemental Figure 4 ; the $\chi^{2}$ test was applied for Figure 6A and Supplemental Figure 3 (upper panels). All quantifications were performed on more than 3 independent experiments to account for technical variability. The size of the population $(n)$ is provided in each figure legend. As presented in the figures, the differences between experimental groups are significant for ${ }^{*} P<0.05$; ${ }^{* *} P<0.01,{ }^{* *} P<0.001$, and ${ }^{* * * *} P<0.0001$.

Study approval. Experiments on zebrafish were conducted prior to 5 days after fertilization, which corresponds to the nonautonomous stage of the animals and does not require specific authorization accordingly to Directive 2010/63/EU. We obtained the approval of the ethical committee and the French ministry (reference $\mathrm{N}^{\circ} \mathrm{036}$ ) for the creation of the new zebrafish line. 


\section{Author contributions}

PB conceptualized and supervised the study. KSM and YA performed most of the experiments. LL conducted the experiments concerning the genetic gan line. $\mathrm{AB}$ determined the spatiotemporal expression of $\mathrm{z}$-gigaxonin. $\mathrm{M}$ Ruiz performed the bioinformatics analysis and the rescue experiments. M Rossel helped with scientific discussions. $\mathrm{PB}$ wrote the manuscript, with contribution from KSM, YA, and LL. PB reviewed and edited the manuscript. First authorship was determined by alphabetical order.

\section{Acknowledgments}

We are grateful to P. Beachy for providing the Shh Light-2 and ShhN cell lines, and to A. Schier and P. Huang for the ptch2 probe. We thank C. Conan for formatting the movies on zebrafish; the CRBM zebrafish facility (Montpellier) and B. Delaval for the coordination of housing and support; P. Richard, M. Plays, and N. Cubedo for zebrafish husbandry and assistance in injection; the MRI-COMET facility (C. Cazevieille, Montpellier) for electron microscopy; Y. Talmat-Amar for assisting in the preparation of samples for electron microscopy; and S. Venteo for advice on in situ hybridization. We are also grateful to the IGF Institute (Montpellier) and especially C. Joplin and A. Faucherre for the provision of the Zebrabox, Carl Zeiss for the LightSheet Z.1 microscope, and N. Tricaud for the confocal laser-scanning microscope. This work was funded by l'Institut
National de la Santé et de la Recherche Médicale to PB, l'Association Française contre les Myopathies (AFM) to YA (fellowship 17721), and the Fondation pour la Recherche Médicale to KSM (fellowship SPF20160936324), LL (fellowship PLP20170939065), and AB (fellowship ING20111223593). PB is supported by grants from the ATIP-Avenir program (INSERM), the AFM (grant 17654), and the Fondation Maladies Rares (FMR). We are very grateful to J. Hazan for scientific discussions; S. Schneider-Maunoury, H. Gilbreath, P. Carroll, and G. Campbell for critical reading and comments on the manuscript; and K. Loulier and F. Michon for helpful discussion during the revision.

Address correspondence to: Pascale Bomont, INM-INSERM U1051, Hopital St Eloi, 80 Rue A. Fliche BP 74103, 34091 Montpellier, France. Phone: 33.4.99.63.60.73; Email: pascale.bomont@inserm.fr.

YA's present address is: UNIL-DP, Bugnon 7, 1005 Lausanne, Switzerland.

AB's present address is: EDM-CHUV, Bugnon 07, 1005 Lausanne, Switzerland.

M Ruiz's present address is: JSEI, UCLA, Los Angeles, California, USA.
1. DiFiglia M, et al. Huntingtin is a cytoplasmic protein associated with vesicles in human and rat brain neurons. Neuron. 1995;14(5):1075-1081.

2. Kimberly WT, Zheng JB, Town T, Flavell RA, Selkoe DJ. Physiological regulation of the betaamyloid precursor protein signaling domain by c-Jun N-terminal kinase JNK3 during neuronal differentiation. J Neurosci. 2005;25(23):5533-5543.

3. Lorent K, Overbergh L, Moechars D, De Strooper B, Van Leuven F, Van den Berghe H. Expression in mouse embryos and in adult mouse brain of three members of the amyloid precursor protein family, of the alpha-2-macroglobulin receptor/ low density lipoprotein receptor-related protein and of its ligands apolipoprotein E, lipoprotein lipase, alpha-2-macroglobulin and the 40,000 molecular weight receptor-associated protein. Neuroscience. 1995;65(4):1009-1025.

4. Paulsen JS, et al. Brain structure in preclinical Huntington's disease. Biol Psychiatry. 2006;59(1):57-63.

5. Reiman EM, et al. Brain imaging and fluid biomarker analysis in young adults at genetic risk for autosomal dominant Alzheimer's disease in the presenilin 1 E280A kindred: a case-control study. Lancet Neurol. 2012;11(12):1048-1056.

6. Milnerwood AJ, Raymond LA. Corticostriatal synaptic function in mouse models of Huntington's disease: early effects of huntingtin repeat length and protein load. J Physiol (Lond). 2007;585(Pt 3):817-831.

7. Young-Pearse TL, Bai J, Chang R, Zheng JB, LoTurco JJ, Selkoe DJ. A critical function for beta-amyloid precursor protein in neuronal migration revealed by in utero RNA interference. JNeurosci. 2007;27(52):14459-14469.

8. Liu H, Shafey D, Moores JN, Kothary R. Neurodevelopmental consequences of Smn depletion in a mouse model of spinal muscular atrophy. J Neurosci Res. 2010;88(1):111-122.

9. Molero AE, Gokhan S, Gonzalez S, Feig JL, Alexandre LC, Mehler MF. Impairment of developmental stem cell-mediated striatal neurogenesis and pluripotency genes in a knock-in model of Huntington's disease. Proc Natl Acad Sci USA. 2009;106(51):21900-21905.

10. Molina-Calavita M, Barnat M, Elias S, Aparicio E, Piel M, Humbert S. Mutant huntingtin affects cortical progenitor cell division and development of the mouse neocortex. JNeurosci. 2014;34(30):10034-10040.

11. Molero AE, et al. Selective expression of mutant huntingtin during development recapitulates characteristic features of Huntington's disease. Proc Natl Acad Sci USA. 2016;113(20):5736-5741.

12. Berg BO, Rosenberg SH, Asbury AK. Giant axonal neuropathy. Pediatrics. 1972;49(6):894-899.

13. Johnson-Kerner BL, Roth L, Greene JP, Wichterle $\mathrm{H}$, Sproule DM. Giant axonal neuropathy: An updated perspective on its pathology and pathogenesis. Muscle Nerve. 2014;50(4):467-476.

14. Kuhlenbaumer G, Timmerman V, Bomont P. Giant axonal neuropathy. In: Adam MP, et al, eds GeneReviews [Internet]. Seattle (WA): University of Washington, Seattle; 1993-2019.

15 . Bomont $\mathrm{P}$, et al. The gene encoding gigaxonin, a new member of the cytoskeletal BTB/kelch repeat family, is mutated in giant axonal neuropathy. Nat Genet. 2000;26(3):370-374.

16. Demir E, et al. Giant axonal neuropathy: clinical and genetic study in six cases. J Neurol Neurosurg Psychiatry. 2005;76(6):825-832.

17. Ganay T, Boizot A, Burrer R, Chauvin JP, Bomont P. Sensory-motor deficits and neurofilament disorganization in gigaxonin-null mice. $\mathrm{Mol} \mathrm{Neu}$ rodegener. 2011;6:25.
18. Furukawa M, He YJ, Borchers C, Xiong Y. Targeting of protein ubiquitination by BTBCullin 3-Roc1 ubiquitin ligases. Nat Cell Biol. 2003;5(11):1001-1007.

19. Boizot A, et al. The instability of the BTB-KELCH protein gigaxonin causes giant axonal neuropathy and constitutes a new penetrant and specific diagnostic test. Acta Neuropathol Commun. 2014;2:47.

20. Bomont P. Degradation of the intermediate filament family by gigaxonin. Meth Enzymol. 2016;569:215-231.

21. Prineas JW, Ouvrier RA, Wright RG, Walsh JC, McLeod JG. Gian axonal neuropathy--a generalized disorder of cytoplasmic microfilament formation. J Neuropathol Exp Neurol. 1976;35(4):458-470.

22. Dequen F, Bomont P, Gowing G, Cleveland DW, Julien JP. Modest loss of peripheral axons, muscle atrophy and formation of brain inclusions in mice with targeted deletion of gigaxonin exon 1. J Neurochem. 2008;107(1):253-264.

23. Israeli $\mathrm{E}$, et al. Intermediate filament aggregates cause mitochondrial dysmotility and increase energy demands in giant axonal neuropathy. Hum Mol Genet. 2016;25(11):2143-2157.

24. Mahammad S, et al. Giant axonal neuropathyassociated gigaxonin mutations impair intermediate filament protein degradation. J Clin Invest. 2013;123(5):1964-1975.

25. Bomont $P$, Koenig M. Intermediate filament aggregation in fibroblasts of giant axonal neuropathy patients is aggravated in non dividing cells and by microtubule destabilization. $\mathrm{Hum} \mathrm{Mol}$ Genet. 2003;12(8):813-822.

26. Cleveland DW, Yamanaka K, Bomont P. Gigaxonin controls vimentin organization through a tubulin chaperone-independent pathway. Hum Mol Genet. 2009;18(8):1384-1394. 
27. Johnson-Kerner BL, et al. Intermediate filament protein accumulation in motor neurons derived from giant axonal neuropathy iPSCs rescued by restoration of gigaxonin. Hum Mol Genet. 2015;24(5):1420-1431.

28. Scrivo A, Codogno P, Bomont P. Gigaxonin E3 ligase governs ATG16L1 turnover to control autophagosome production. Nat Commun. 2019;10(1):780.

29. Ingham PW, McMahon AP. Hedgehog signaling in animal development: paradigms and principles. Genes Dev. 2001;15(23):3059-3087.

30. Jessell TM. Neuronal specification in the spinal cord: inductive signals and transcriptional codes. Nat Rev Genet. 2000;1(1):20-29.

31. Te KG, Reggiani C. Skeletal muscle fibre type specification during embryonic development. JMuscle Res Cell Motil. 2002;23(1):65-69.

32. Bale AE. Hedgehog signaling and human disease. Annu Rev Genomics Hum Genet. 2002;3:47-65.

33. Jiang J, Hui CC. Hedgehog signaling in development and cancer. Dev Cell. 2008;15(6):801-812.

34. Briscoe J, Thérond PP. The mechanisms of Hedgehog signalling and its roles in development and disease. Nat Rev Mol Cell Biol. 2013;14(7):416-429.

35. Wheway G, Nazlamova L, Hancock JT. Signaling through the Primary Cilium. Front Cell Dev Biol. 2018;6:8.

36. Haycraft CJ, Banizs B, Aydin-Son Y, Zhang Q, Michaud EJ, Yoder BK. Gli2 and Gli3 localize to cilia and require the intraflagellar transport protein polaris for processing and function. PLOS Genet. 2005;1(4):e53.

37. Huangfu D, Anderson KV. Cilia and Hedgehog responsiveness in the mouse. Proc Natl Acad Sci USA. 2005;102(32):11325-11330.

38. Jiang J. Regulation of Hh/Gli signaling by dual ubiquitin pathways. Cell Cycle. 2006;5(21):2457-2463.

39. Li T, et al. Ubr3, a novel modulator of Hh signaling affects the degradation of Costal-2 and Kif7 through poly-ubiquitination. PLoS Genet. 2016;12(5):e1006054.

40. Schweiger S, et al. The E3 ubiquitin ligase MID1 catalyzes ubiquitination and cleavage of Fu. J Biol
Chem. 2014;289(46):31805-31817.

41. Zhou Z, et al. Deubiquitination of Ci/Gli by Usp7/ HAUSP Regulates Hedgehog Signaling. Dev Cell. 2015;34(1):58-72.

42. Huang S, et al. Activation of Smurf E3 ligase promoted by smoothened regulates hedgehog signaling through targeting patched turnover. PLoS Biol. 2013;11(11):e1001721.

43. Yue S, et al. Requirement of Smurf-mediated endocytosis of Patched 1 in sonic hedgehog signal reception. Elife. 2014;3:e02555.

44. Chen XL, et al. Patched-1 proapoptotic activity is downregulated by modification of K1413 by the E3 ubiquitin-protein ligase Itchy homolog. Mol Cell Biol. 2014;34(20):3855-3866.

45. Lewis KE, Eisen JS. From cells to circuits: development of the zebrafish spinal cord. Prog Neurobiol. 2003;69(6):419-449.

46. Chen W, Burgess S, Hopkins N. Analysis of the zebrafish smoothened mutant reveals conserved and divergent functions of hedgehog activity. Development. 2001;128(12):2385-2396.

47. Vanderlaan G, Tyurina OV, Karlstrom RO, Chandrasekhar A. Gli function is essential for motor neuron induction in zebrafish. Dev Biol. 2005;282(2):550-570.

48. Varga ZM, et al. Zebrafish smoothened functions in ventral neural tube specification and axon tract formation. Development. 2001;128(18):3497-3509.

49. Buysse K, et al. Giant axonal neuropathy caused by compound heterozygosity for a maternally inherited microdeletion and a paternal mutation within the GAN gene. Am JMed Genet $A$. 2010;152A(11):2802-2804.

50. Karlstrom RO, et al. Genetic analysis of zebrafish gli1 and gli2 reveals divergent requirements for gli genes in vertebrate development. Development. 2003;130(8):1549-1564.

51. Taipale J, et al. Effects of oncogenic mutations in Smoothened and Patched can be reversed by cyclopamine. Nature. 2000;406(6799):1005-1009.

52. Corbit KC, Aanstad P, Singla V, Norman AR, Stainier DY, Reiter JF. Vertebrate Smoothened functions at the primary cilium. Nature.
2005;437(7061):1018-1021.

53. Beattie CE, Hatta K, Halpern ME, Liu H, Eisen JS, Kimmel CB. Temporal separation in the specification of primary and secondary motoneurons in zebrafish. Dev Biol. 1997;187(2):171-182.

54. Avilés EC, Wilson NH, Stoeckli ET. Sonic hedgehog and Wnt: antagonists in morphogenesis but collaborators in axon guidance. Front Cell Neurosci. 2013;7:86.

55. Lee RT, Zhao Z, Ingham PW. Hedgehog signalling. Development. 2016;143(3):367-372.

56. De Mori R, et al. Hypomorphic recessive variants in SUFU impair the Sonic Hedgehog pathway and cause Joubert Syndrome with craniofacial and skeletal defects. Am J Hum Genet. 2017;101(4):552-563.

57. Schimmenti LA, et al. Novel mutation in sonic hedgehog in non-syndromic colobomatous microphthalmia. Am JMed Genet A. 2003;116A(3):215-221.

58. Roessler E, et al. Mutations in the human Sonic Hedgehog gene cause holoprosencephaly. Nat Genet. 1996;14(3):357-360.

59. Rubino S, Qian J, Pinheiro-Neto CD, Kenning TJ, Adamo MA. A familial syndrome of hypothalamic hamartomas, polydactyly, and SMO mutations: a clinical report of 2 cases. J Neurosurg Pediatr. 2018;23(1):98-103.

60. Chen SD, Yang JL, Hwang WC, Yang DI. Emerging roles of Sonic Hedgehog in adult neurological diseases: neurogenesis and beyond. Int J Mol Sci. 2018;19(8):E2423.

61. Traiffort E, Angot E, Ruat M. Sonic Hedgehog signaling in the mammalian brain. JNeurochem. 2010;113(3):576-590.

62. Haeussler M, et al. Evaluation of off-target and on-target scoring algorithms and integration into the guide RNA selection tool CRISPOR. Genome Biol. 2016;17(1):148.

63. Angebault C, et al. Recessive mutations in RTN4IP1 cause isolated and syndromic optic neuropathies. Am JHum Genet. 2015;97(5):754-760.

64. Zhao C. Hedgehog (Hh) reporter activity assay. Bio-Protoc. 2014;4(14):e1182. 